\title{
Voluntary Provision of Communities for the Provision of Public Projects
}

\author{
Ori Haimanko*, Michel Le Breton†, and Shlomo Weber ${ }^{\ddagger}$
}

February 14, 2003

\begin{abstract}
In this paper we examine a collective decision problem, where the set of heterogeneous individuals is partitioned into several groups, each choosing its own policy (e.g., location of a public project) from the given policy space. The model is that of "horizontal product differentiation" where individuals display distinct preferences over the policy space. We first consider the notion of "efficient" partition that minimizes the total policy-related costs and aggregate personalized costs. (The latter are incurred when an individual belongs to a group that does not choose her most preferred, ideal, policy.) We then examine "sustainable" partitions, in which the policy-related costs can be distributed in a way that no subgroup (belonging to the partition or not) has an incentive to break away from the rest and to set its own policy. Our main result is that, with a unidimensional policy space and single-peaked personalized costs, every efficient partition is sustainable. We further describe some important features of efficiency by characterizing the efficient distribution (and number) of policies chosen from the policy space when their cost is small. It turns out that efficiency is achieved when the distribution of policies follows the square root of the density of individuals' ideal choices.
\end{abstract}

*Ben-Gurion University, Beer-Sheva, Israel.

†Université de la Méditérannée, Marseille, France.

‡Southern Methodist University, Dallas, USA. 


\section{Introduction}

Consider a group of heterogeneous agents that display different preferences over the given set of feasible policies $I$. The agents can act as a united group and choose only one policy from the set $I$, or alternatively, they may partition themselves into several groups, each making its own selection from $I$. Since each policy carries monetary cost, increasing returns to scale of large groups generate advantages for the "grand coalition" of all agents that reduces a per capita contribution towards the monetary cost of implementing that policy. On the other hand, the heterogeneity of preferences within a large group may create a situation where some agents face a policy far away from their most preferred choice. In this case these agents face high "personalized costs," that grow with the "distance" between the chosen policy and their ideal choice. It could be possible, therefore, that a subgroup of dissatisfied agents would consider a break-up from the rest of the group, and selection of a different policy that better fits the preferences of its members and reduces their personalized costs. Thus, low costs of the policy implementation could make a multi-group configuration more efficient than the grand coalition, the formation of which is unlikely in many cases. It is important to stress again that this phenomena is due to "horizontal product differentiation" that, in our model, amounts to heterogeneity of agents' preferences over the policy. In contrast, a multi-group configuration would not arise in the traditional public good problem without congestion effects, where public goods are "vertically differentiated", and all agents exhibit identical preferences based on either quantity or quality attributes of public goods.

The aforementioned conflict between increasing returns to scale and heterogeneity of agents' preferences arises in many different contexts. For example, a large political party, as compared to a small one, may provide a higher level of material, political or career benefits for its members. However, the political platforms chosen by the large party could create a dissent among substantial number of party members who would prefer a different policy choice, and may even prompt some of them to leave the party. In an economy where the local public goods are produced by means of either proportional or poll tax, a large jurisdiction 
may lessen the agents' tax burden. However the choice of public policy or amount of public goods produced within the jurisdiction could be undesirable for some of its residents. Indeed, a substantial investment in public schools may be undesirable for childless families or agents. Another important example of conflict between the size of the group and the population heterogeneity arises in the context of the country formation when a region of a country may contemplate a secession if the gap between monetary, ethnic, cultural or religious policies of the central government and the regional expectations and aspirations becomes too large.

In order to formally examine the outcomes of the decision-making process, whether the grand coalition or a multi-group configuration, we first address the issue of their "efficiency". Every partition of agents and choice of the corresponding policies generates two cost components: the total monetary cost of chosen policies and the aggregate personalized costs. Note that the first cost component rises with the number of selected policies, whereas the second component decreases with the number of policies. When the preferences of the agents are quasi-linear with respect to some numeraire, i.e., if utility is transferable across agents, a collective decision is Pareto efficient if it minimizes the total cost generated by the decision.

The description of the collective decision problem is still incomplete since it provides no mechanism of sharing the policy costs among agents choosing the same policy. (Indeed, a government must know how to tax its citizens to finance implementation of public policies. A jurisdiction must as well decide on the tax burden of its residents for financing a new public project, etc.) In other words, when the efficient partition of agents has formed and a set of policies has been chosen, the allocation of the cost among the agents should be determined. For an arbitrarily chosen cost allocation, however, there may exist a group $C$ of agents that can reject the proposed collective arrangement by choosing a policy and a device for financing it such that everybody in $C$ would be better off compared to the current arrangement. Our main purpose is to examine those collective decisions and corresponding cost allocations that are "sustainable" in the sense that no group can reject a proposed arrangement. The sustainability requirement serves as the group-participation constraint, which is of particular importance in absence of a strong central authority capable of enforcing a collective arrangement against the 
wishes of some agents.

The sustainability notion employed here is quite strong. We allow for every group of agents to pose a threat to proposed arrangement. When the partition of agents and their policies are determined, the arrangement should be immune against the threats from "inside", i.e., no group choosing the same policy can suggest an alternative policy choice that will make every member of the group better off. The immunity should also be from "outside" threats, i.e., no group that consists of agents originally choosing different policies can make its members better off by uniting. Both the "inside" and the "outside" threats can be made by groups that are not in any way related to the original partition. Thus, the sustainability requirement imposes very stringent constraints on the selection of the financing device. It is not surprising, therefore, that one has to impose some restrictions in order to guarantee the existence of sustainable allocations. We demonstrate that the unidimensionality of set of policies and single-peakedness of personalized agents' costs over the policy space not only guarantee the existence of a sustainable partition, but also yields the equivalence of efficient and sustainable partitions. That is, every efficient partition is also sustainable!

On the formal level, our model uses the notion of public project (Mas-Colell (1980)). It appears in many different settings and can accommodate virtually any interpretation, as long as the benefit derived by an agent from the project is not affected by the size and the composition of the group of other agents who are assigned to the same project, i.e., the public project is pure in the traditional sense. In particular, the problem of choosing public policies that we discussed earlier can be restated using the new terminology (i.e., $I$ is now the set of feasible public projects, from which groups of agents have to make choices), without any loss of generality.

The description of a public project may involve many characteristics including notably its location. If projects are public facilities (hospitals, swimming-pools, libraries, etc.) and location is the parameter of horizontal differentiation, then the personalized costs to which we referred above are simply the transportation costs to projects' locations ${ }^{1}$. The cost of every

\footnotetext{
${ }^{1}$ The transportation costs leads to private costly access as defined by Cremer and Laffont (2000) but they
} 
project depends on the number of agents assigned to it (users) and is assumed to consist of the project-independent fixed cost and the variable cost that increases with the number of users. As we indicated above, we impose two major assumptions: as in the traditional Hotelling model, the parameter of horizontal differentiation of the policy space is unidimensional, and the personalized (transportation) costs are single-peaked in the policy space. In all other aspects our model is very general and we allow for arbitrary populations of agents ranging from atomic sets to atomless probability distributions to general distributions. As was said, our main result yields the equivalence of the sets of efficient and sustainable partitions.

We also examine the number of projects determined by efficient partitions within any given subset of the policy space. By identifying every agent with her most preferred project, we establish the simple relationship between the population density on any given subset $J$ of the policy space and the efficient number of projects assigned to $J$ and prove that the asymptotic density of efficiently located projects is proportional to the square root of the population density. The square root stresses the equalizing effect of efficiently distributed public projects on the agents: subsets $J$ with a low population density typically get more than their "fair share" of public projects on the expense of areas with high population density. In particular, it may happen that a subset with a smaller population will receive a larger number of projects than a subset with a larger population.

The paper is organized as follows. After the review of related literature, we present the model and state our main result on equivalence of efficiency and sustainability. In Section 3 we examine the number of projects supported by efficient partitions. In addition, we establish the link between the population density and the number of projects in an efficient partition within any given subset of the policy space. In Section 4 we prove our main result. Proofs of other results are relegated to the Appendix.

\section{Related Literature}

This paper lies at the crossroads of many different fields: combinatorial optimization, coassume that the private access cost of a customer does not depend upon the public project to which she is assigned. 
operative games with a continuum of players, theoretical public finance and political economy, and will describe the relationship of our work with these different topics.

The analysis of cost minimization in uncapacitated facility location problems, akin to those arising in the context of horizontally differentiated public projects, even though the facility location problems face exogenously given (and finite) number of choices rather than endogenous set of locations. It has become a major topic of research in operations research and combinatorial optimization. After noticing that facility location problem can be expressed as a linear-integer problem (Balinski (1965)), the emphasis has been on the search of efficient algorithms to compute the solution to this problem, or, at least, its approximation. This literature is surveyed in Cornuejols, Nemhauser and Wolsey (1990) and is still a subject of very active research.

Although the operations research literature has primarily focused on the algorithmic aspects of cost minimization problems, some recent contributions address the issue of efficient cost allocations. Existence of the core have received some attention and is the subject of several contributions (Goemans and Skutella (2000), Grishukhin (1994), Kolen (1983), Kolen and Tamir (1990), Tamir (1992), Trubin (1976)). The first result shows that the core of the cost allocation game induced by a facility location problem is nonempty if and only if there is no integrality gap, i.e., if the value of the linear-integer program is the same as the value of the program where the integer constraints have been relaxed to linear constraints. Using this result, Goemans and Skutella (2000) show that there is no integrality gap when transportation costs are single peaked over the unidimensional set of feasible locations, thus yielding the nonempty core in this case. However, these contributions assume the finiteness of the sets of agents and exogenously given facilities whereas we consider any type of distributions and an endogenous choice of policies as we believe that many environments are better represented by this framework.

The literature on cooperative games with a continuum of players has mostly focused on the Shapley value, originally defined only for games with a finite number of players (Aumann and Shapley (1974)). There are few papers on the core of cooperative games with a continuum of 
players (Ichiishi and Weber (1978), Kannai (1969), Schmeidler (1967), Weber (1981), Wooders (1983), Wooders and Zame (1984)). This literature, surveyed in Kannai (1992), has mostly focused on the appropriate extension of the notion of balancedness for games with a continuum of players. Here, we do not use balancedness and instead we exploit the specific nature of our game and perform a limit argument. There is a more recent literature on the core of games with a continuum of players (Einy, Moreno and Shitovitz (1999), Epstein and Marinnaci (2001)), but the auxiliary games that we consider do not satisfy their conditions, and so the proofs must take a different route.

Finally, our paper relates to the literature on the formation of jurisdictions which is also based on a trade-off between the heterogeneity of citizens' preferences across and economies of scale (Casella (1992), Cremer, De Kerchove and Thisse (1885), Greenberg and Weber (1986), Guesnerie and Oddou (1981)(1987), Jéhiel and Scotchmer (2001), Konishi, Le Breton and Weber (1998), Weber and Zamir (1985), Westhoff (1977), Wooders (1978)). There is also a more recent literature on the political economy of the process of country formation (Alesina and Spolaore (1997), Alesina, Spolaore and Wacziarg (2000), Bolton, Spolaore and Roland (1996), Wei (1991)) where the threat of secession and its impact of the equilibrium configuration is explicitly considered. In many of these papers the world population is described through a continuous distribution but except for Haimanko, Le Breton and Weber (2001) and Le Breton and Weber (1999), the role of transfers to deter secession threats is not very much investigated. Haimanko, Le Breton and Weber (2001) demonstrate the necessity of transfers for polarized distributions while Le Breton and Weber (2000) offer a partial characterization of the transfer mechanisms.

\section{The Model}

We consider a society that consists of agents with preferences over the set $I$ of feasible locations of public projects. The set $I$ is assumed to be unidimensional and is given by the interval $[0,1]$. Every agent $t$ has an ideal point in $I$ and the preferences that are symmetric 
and single-picked, and we identify $t$ with her ideal point in $I$. The distribution of ideal points is given by a cumulative distribution function $F$, defined over the space $I$. It is important to stress that we do not impose any restrictions on the distribution function and even allow for "atoms", i.e., for positive masses of agents to be located at the same point of $I$. The only property of $F$ we need is its monotonicity on the interval $[0,1]$. We denote by $\Lambda$ the measure on $I$ induced by the distribution function $F$ with the total mass $\Lambda(I)$ equal to 1 .

A subset of $I$ which is a union of a finite number of intervals will be called a community. For notational and analytical simplicity, we will restrict our attentions to communities when subsets of $I$ will be involved.

The costs associated with any project $p$ must be covered by the community $C \subset I$ of its users. The cost of the project, given by

$$
G(p, C)=g(p)+\alpha \Lambda(C)
$$

consists of two components: the positive fixed cost of setting and maintaining the project, $g(p)$, and the variable cost that depends on the number of project users, $\alpha \cdot \Lambda(C)$, where $\alpha$ ia nonnegative constant. We assume ${ }^{2}$ that the fixed cost of all projects is the same:

Fixed Cost Invariance: $g(p)=g$ for all projects $p \in I$.

This assumption implies that for a given group of users $C$, the project $\operatorname{cost} G(p, C)$ is independent of a choice of the project $p$. Thus, we can simply use the notation $G(C)$ instead of $G(p, C)$.

If an agent $t$ resides in community $C$ that uses project $p \in I$, the disutility or "transportation" cost incurred by $t, d(t, p)$, is determined by the distance between $t$ and the location $p$ of the project. We only require that

Continuity and Monotonicity of Transportation Costs: The cost function $d(t, p)=d(\mid t-$ $p \mid$ ) is continuous and (strictly) increasing in the distance $|t-p|$, with $d(0)=0$.

\footnotetext{
${ }^{2}$ Our main result remains intact even if this assumption is weakened, by assuming that $g(p)$ is a continuous and positive-valued function of $p$. Fixed cost invariance assumption will nevertheless be maintained, for the sake of convenience and increased clarity of proofs.
} 
If only one project serves the entire population, it could be far away from significant fraction of agents whose ideal points are located close to one of the margins. Thus, in order to examine efficiency and sustainability of the set of chosen projects, it is natural to consider a multi-project setting. Let $\mathcal{P}$ be a (nonempty) finite ordered set $\left(p_{1}, \ldots, p_{K}\right)$ of projects, and $\pi$ is an ordered partition of $I$ into communities $\left(C_{1}, C_{2}, \ldots, C_{K}\right)$, where the community $C_{k}$ represents the set of users of the project $p_{k}$. Every two different communities $C_{k}, C_{l} \in \pi$, have an empty intersection, and the union of all communities in $\pi$ covers the entire set $I$, i.e., each agent $t \in I$ belongs to one and only one community in $\pi$. For notational and analytic simplicity, we restrict our examination only to communities that consist of a finite number of intervals.

Every pair $(\mathcal{P}, \pi)$, where the set of projects $\mathcal{P}$ and the partition $\pi$ have the same number of elements, will be referred to as project-user configuration (or PUC). An important class of PUC consists of pairs $(\mathcal{P}, \pi)$, where each community $C \in \pi$ is a convex set, or, simply, an interval. In this case communities of users satisfy the connectedness property: for every three users, $t<t^{\prime}<t^{\prime \prime}$, whenever $t$ and $t^{\prime \prime}$ belong to the same community $C$, the user $t^{\prime}$ must belong to $C$ as well. Such partitions $\pi$ will be called "connected".

For every PUC $(\mathcal{P}, \pi)$, we shall denote by $T(\mathcal{P}, \pi)$ the aggregate (transportation and project) cost incurred by PUC $(\mathcal{P}, \pi)$ :

$$
T(\mathcal{P}, \pi)=\sum_{k=1}^{K}\left[D\left(p_{k}, C_{k}\right)+G\left(C_{k}\right)\right],
$$

where for every $k$, the aggregate transportation cost of community $C_{k}$ served by project $p_{k}$, $D\left(p_{k}, C_{k}\right)$, is given by

$$
D\left(p_{k}, C_{k}\right)=\int_{C_{k}} d\left(\left|t-p_{k}\right|\right) d \Lambda(t) .
$$

For every community $C$ we denote by $D(C)$ the minimal transportation cost within $C$ :

$$
D(C)=\inf _{p \in I} \int_{C} d(|t-p|) d \Lambda(t) .
$$

We shall show that the set of projects $p$, at which this infimum is attained, is nonempty. Every such project $p$ will be called $C$-optimal. 
We are now in a position to introduce the notion of efficient PUC:

Definition: A PUC $(\mathcal{P}, \pi)$ is called efficient if it minimizes the aggregate cost over all PUC, i.e., $T(\mathcal{P}, \pi) \leq T\left(\mathcal{P}^{\prime}, \pi^{\prime}\right)$ for any $\left(\mathcal{P}^{\prime}, \pi^{\prime}\right)$. If $(\mathcal{P}, \pi)$ is an efficient PUC, $\mathcal{P}$ will be called an efficient set of projects, and $\pi$ will be called an efficient partition.

This definition immediately implies that in every efficient partition, every community chooses its optimal project:

Remark 2.1: Let $\operatorname{PUC}(\mathcal{P}, \pi)$, where $\mathcal{P}=\left(p_{1}, \ldots, p_{K}\right)$ is the set of projects, and $\pi=$ $\left(C_{1}, C_{2}, \ldots, C_{K}\right)$ is the set of corresponding communities, be efficient. Then for every $k=1, \ldots, K$, the project $p_{k}$ is $C_{k}$-optimal.

Our first result states that an efficient PUC always exists. Moreover, there exists an efficient PUC $(\mathcal{P}, \pi)$ such that every $C \in \pi$ is an interval:

Proposition 2.2: There exist an efficient PUC. Moreover, there is an efficient $\mathrm{PUC}(\mathcal{P}, \pi)$ such that the partition $\pi$ is connected.

The following proposition shows that in many cases the efficient set of projects uniquely (up to inclusion or exclusion of boundary points) determines the corresponding efficient partition, and thus the entire PUC. For this reason, the attention can be sometimes confined only to efficient sets of projects (as will be done, for instance, in the next section).

Proposition 2.3: Let $(\mathcal{P}, \pi)$ be a an efficient PUC such that $\mathcal{P}=\left(p_{1}, \ldots, p_{K}\right)(K \geq 2)$ with $p_{1}<p_{2}<\ldots<p_{K}$. If the distribution of ideal points possesses a continuous and strictly positive density function, then the efficient partition $\pi=\left(I_{1}, \ldots, I_{K}\right)$ is uniquely determined (up to the boundary points of the communities in $\pi$ ) by

$$
I_{1}=\left[0, \frac{p_{1}+p_{2}}{2}\right), \ldots, I_{k}=\left[\frac{p_{k-1}+p_{k}}{2}, \frac{p_{k}+p_{k+1}}{2}\right), \ldots, I_{K}=\left[\frac{p_{K-1}+p_{K}}{2}, 1\right]
$$


This proposition demonstrates that the efficient partition is, in fact, the set of intervals $\left[0, b_{1}\right), \ldots,\left[b_{k}, b_{k+1}\right), \ldots,\left[b_{K-1}, 1\right]$, where every "cut-off" user $b_{k}, k=1, \ldots, K-1$, is equally close to project $p_{k-1}$ on her left and project $p_{k}$ on her right.

By Remark 2.1, every community that constitutes a part of an efficient partition always selects its optimal project. This means that there is no other project that would reduce the total costs of this community. However, it does not rule out a possibility that the efficient configuration would not be stable under a threat of rejection by a group of users that consists of parts of different communities in $\pi$. To address this issue, we must examine how the project costs are allocated within a community of users. The charges that should be imposed on project users in order to cover the project cost cannot be found in, or deduced from, the description of a PUC, and any community $C \in \pi$ has to determine the monetary contribution of each resident $t$ towards the project cost $G(C)$. To formalize the discussion, we introduce the notion of a $C$-cost allocation, which allows for all lump sum transfers within $C$ that satisfy the budget constraint:

Definition: A measurable function $x$ defined on the set $C$ is called a $C$-cost allocation if

$$
\int_{C} x(t) d \Lambda(t)=G(C)
$$

If $(\mathcal{P}, \pi)$ is a PUC, where $\pi=\left(C_{1}, \ldots, C_{K}\right)$, and $x^{k}$ is a $C_{k^{-}}$-cost allocation for $k=$ $1, \ldots, K$, the vector $\bar{x}=\left(x^{1}, \ldots, x^{K}\right)$ will be called a $(\mathcal{P}, \pi)$-cost allocation.

It is natural, and important, to ask whether a given efficient allocation $(\mathcal{P}, \pi)$ is sustainable in the face of possible rejections by groups of users. That is, whether there exists a $(\mathcal{P}, \pi)$-cost allocation $\bar{x}$ such that no set of users $C$ can defy the arrangement dictated by $\bar{x}$, select its own project, and come up with a cost sharing scheme that reduces the total cost incurred by every member of $C$. The existence of non-sustainable efficient PUC would significantly undermine the efficiency appeal, and, if all efficient PUC turn out to be non-sustainable, it would provide a cause for further concern about robustness of our efficiency concept. Fortunately, and surprisingly, sustainability and efficiency turn out to be equivalent concepts. The following definition, which formalizes the notion of sustainability, is followed by our main result. 
Definition: Consider a $\mathrm{PUC}(\mathcal{P}, \pi)$ with $\mathcal{P}=\left(p_{1}\right.$ 
our problem with the cooperative game $V^{\Lambda}$, that assigns the value $V^{\Lambda}(C)$ for every community $C$. We then consider the core of this game, which is the set of measures $\mu$ on $I$, such that for all communities $C \mu(C) \leq V^{\Lambda}(C)$ with equality for the set $I$, i.e., $\mu(I) \leq V^{\Lambda}(I)$.

By using the link between our game and a facility location problem (Goemans and Skutella (2000)) with a finite set of agents and exogenously given locations, we first demonstrate that in the case when the set of agents is a finite "atomic" set, i.e., the measure $\Lambda$ has a finite support, the core of the game $V^{\Lambda}$ is nonempty. We further develop quite extensive machinery in order to apply the approximation arguments and to extend the nonemptiness of the core $V^{\Lambda}$ to the class of general measures $\Lambda$. Finally, we pick a measure $\mu$ from the (nonempty) core of the game $V^{\Lambda}$ and slightly perturb $\mu$ in order to obtain a measure $\eta$ that satisfies two desirable properties: it belongs to the core of $V^{\Lambda}$ and is absolutely continuous with respect to $\Lambda$. Then, by applying the Radon-Nykodim Theorem, we explicitly assign a cost share to every agent in such away that guarantee sustainability of the given efficient PUC.

Proposition 2.2 and Main Result imply the existence of a connected sustainable partition. We would like to point out that our problem does not belong to the class of "consecutive games" (Greenberg and Weber (1986), Demange (1994)), where financing devices are restricted in such a way that only consecutive (or connected) coalitions may pose a threat of rejection. In our framework it would simply imply that if an arrangement is rejected by some community $C$, there is an interval $J$ that would also reject this arrangement. Since here we do not a priori restrict the set of possible financing devices, we cannot ignore a possible threat of rejection by disconnected groups of agents. Thus, we cannot use the general combinatorial arguments used to prove the nonemptiness of the core in consecutive games (Greenberg and Weber (1986) and Le Breton, Owen and Weber (1992)).

\section{Quantitative Aspects of Efficiency}

The results of the previous section state that an efficient PUC always exists, and that every efficient PUC can be supported by a suitable cost allocation scheme. It is natural to 
take the next step and to inquire more precisely into the structure of efficient PUC. In this section we examine the number of projects that can be supported by an efficient PUC and the distribution of projects over the interval $I$. More specifically, we derive the relationship between the mass and density of agents on a subset $C$ of $I$ with the number of projects assigned to $C$ by an efficient configuration.

In order to deal with these issues, we assume throughout this section that

Assumption A: The distribution $F$ possesses a strictly positive and continuous density function $f$.

Furthermore, we adopt an asymptotic framework and consider a society where the number of projects in the efficient set is large. All the parameters of the model, except the fixed cost component $g$, will be kept unchanged, so we can focus on the impact of the fixed costs by examining the situation where the value of $g$ tends to zero. Resorting to asymptotic analysis helps to avoid the need to explicitly describe the efficient PUC, which is an unreasonably difficult task for a general distribution function. ${ }^{3}$

The results obtained in this framework are quite sharp. For every interval $J \subset I$ we will be able to assess the number of projects from the efficient set located in $J$, and the precision of our assessment will rise with the enlargement of the efficient set which is achieved by reduction of the project costs. Furthermore, the formula that we develop exhibits a tight and simple link between the limiting distribution of projects in the efficient set and the distribution of population, thus allowing simple comparisons of the numbers of projects efficiently allocated to different intervals.

Formally, consider a subinterval $J$ of $I$. For every positive level of fixed costs $g$, choose an efficient PUC $\left(\mathcal{P}_{g}, \pi_{g}\right)$ and denote by $K_{J}(g)$ the number of projects in $\mathcal{P}_{g}$ located in $J$. The first result of this section describes the asymptotic behavior of $K_{J}(g)$ as $g$ falls to zero, revealing the limiting distribution of the projects from the efficient set over $I$ (and does not depend on a particular chosen family of efficient PUC $\left.\left\{\left(\mathcal{P}_{g}, \pi_{g}\right)\right\}_{g>0}\right)$ :

\footnotetext{
${ }^{3}$ In a special case where the distribution of agents' preferences is uniform, an efficient PUC has been examined in Alesina and Spolaore (1997).
} 
Proposition 3.1: Suppose that the distance function $d$ has the right hand derivative at zero, $d^{\prime}(0)$. Then for every interval $J \subset I$,

$$
\lim _{g \rightarrow 0} K_{g}(J) \sqrt{g}=\frac{\sqrt{d^{\prime}(0)}}{2} \int_{J} \sqrt{f(t)} d t .
$$

Note that that within the class of distributions we consider, the uniform distribution would generate the maximal number of projects to be chosen by an efficient PUC.

It is also worth to pointing out that since the asymptotic "density" of efficiently located projects is proportional to the square root of the population density, areas with low population density typically get more than their "fair share" of public projects on the expense of areas with high population density. In particular, it may happen that an interval with a smaller population will have a larger number of projects than an interval with a larger population. Indeed $\int_{J_{1}} f(t) d t<\int_{J_{2}} f(t) d t$ (i.e., $\left.\Lambda\left(J_{1}\right)<\Lambda\left(J_{2}\right)\right)$ could be perfectly consistent with the reverse inequality $\int_{J_{1}} \sqrt{f(t)} d t>\int_{J_{2}} \sqrt{f(t)} d t$.

One important side of efficiency is that, in the limit, it has a strong equalizing effect on everyones' transportation costs. More precisely, regardless of the population density, in the limit the average transportation cost incurred by agents connected to the same project is approximately the same for any efficiently located project:

Proposition 3.2: Suppose again that the function $d$ has a right hand derivative at zero. Let $J \subset I$ be an arbitrary interval and denote by $D_{g}(J)$ the total transportation cost incurred by an efficient PUC $\left(\mathcal{P}_{g}, \pi_{g}\right)$ on agents in $J$. Put $t_{g}(J) \equiv \frac{D_{g}(J)}{K_{g}(J)}$ (the average aggregate transportation cost per project in $J)$. Then $\lim _{g \rightarrow 0} \frac{t_{g}(J)}{g}=1$.

To illustrate the results presented in this section, consider the following example where the density function of agents' ideal points is given by ${ }^{4}$ :

$$
f(t)= \begin{cases}\frac{1}{2} & \text { if } t \in L \equiv\left[0, \frac{1}{2}\right), \\ \frac{3}{2} & \text { if } t \in R \equiv\left[\frac{1}{2}, 1\right] .\end{cases}
$$

\footnotetext{
${ }^{4}$ This density function is discontinuous at the point $\frac{1}{2}$. However, the example can be easily modified to satisfy Assumption A.
} 
Assume also that the distance function $d$ is linear.

It is easy to see that if $J$ is a subinterval of either $L$ or $R$, then a $J$-optimal project is simply the midpoint of $J$. Propositions 3.1 and 2.3 (or Lemma B.1 from the Appendix) now imply that if the value of fixed cost $g$ is sufficiently low, then the efficient set consists of $l(g)$ equidistant projects on the interval $L$ and $r(g)$ equidistant projects on the interval $R$, and

that $\frac{r(g)}{l(g)} \approx \sqrt{3}$. Furthermore, by Proposition 3.2, in the limit the agents in both intervals, $L$ and $R$, incur the same average transportation cost.

It is important to note that no such equalization would have been possible were the projects locations to match the population density and not its square root. If the projects were indeed allocated on the basis of density, the interval $R$ would receive three times more projects then the interval $L$. Since the aggregate cost of transportation to a project incurred by its users community is proportional to the square of the size of the community, it is easy to verify that the average transportation costs to a project in $L$ would be three times higher than to a project in $R$.

\section{Proof of Main Result}

First, we need some notation and definitions. Let $X$ be a Borel subset of $R$ with the topology induced from $R$. Denote by $\mathcal{M}(X)$ the set of (positive) Borel measures and by $M(X)$ the subset of probability measures on $X$. We start with the definitions of a game and its core utilized in this section.

Definition: A game is a real valued set function defined for all (nonempty) communities in I. Given a game $u$, the core of $u$ is defined as the set of all measures $\mu \in \mathcal{M}(I)$ such that $\mu(C) \leq v(C)$ for all communities $C$, and $\mu(I)=v(I)$.

Let a positive constant $g$ and a strictly increasing function $d:[0,1] \rightarrow \Re_{+}$be given. For every community $C$ and measure $\Lambda \in M(I)$ denote:

$$
v(\Lambda, C)=g+D(C)
$$


where, as in Section 2,

$$
D(C)=\inf _{p \in I} \int_{C} d(|t-p|) d \Lambda(t) .
$$

The nonempty set of "optimal" $p$, i.e. those at which the minimum in (5) is attained, will be denoted by $O P(\Lambda, C)$.

For all $\Lambda \in M(I)$ define the game $V^{\Lambda}$ as follows:

$$
V^{\Lambda}(C)=\left\{\begin{array}{cc}
v(\Lambda, C), & \text { if } C \neq I \\
\inf \sum v\left(\Lambda, C_{j}\right), & \text { if } C=I
\end{array}\right.
$$

where the infimum is taken over all partitions $\left(C_{1}, \ldots, C_{K}\right)$ of $I$ into communities.

We are now in position to state Propositions 4.1 and 4.2, the proofs of which are presented in the Appendix. Assuming the validity of these propositions, we then prove our Main Result.

Proposition 4.1: For any $\Lambda \in M(I)$, the game $V^{\Lambda}$ has a nonempty core.

The following result provides us with an absolutely continuous measure in the core. To recall, a measure $\lambda$ is absolutely continuos with respect to measure $\Lambda$, or $\Lambda$-absolutely continuous, if $\lambda(C)=0$ whenever $\Lambda(C)=0$.

Proposition 4.2: For any $\Lambda \in M(I)$, the core of the game $V^{\Lambda}$ contains a measure which is absolutely continuous with respect to $\Lambda$.

The Radon-Nikodym Theorem (Halmos (1988), Theorem B) implies that for any measure $\mu$, which is $\Lambda$ - absolutely continuous, there is a $\Lambda$-integrable function $\zeta$, called the RadonNikodym derivative of $\mu$, such that for every $C$

$$
\mu(C)=\int_{C} \zeta(t) d \Lambda(t)
$$

We shall call $\zeta$ a core allocation of the game $V^{\Lambda}$ if the corresponding measure $\mu$ belongs to the core of $V^{\Lambda}$. In other words, $\zeta$ is a core allocation of $V^{\Lambda}$ if and only if it is a Radon-Nikodym "derivative" with respect to $\Lambda$ of some measure in the core of $V^{\Lambda}$. Proposition 4.2 yields:

Corollary 4.3: For any $\Lambda \in M(I)$ the set of core allocations of $V^{\Lambda}$ is nonempty. 
Proof of Main Result: We will show first that any efficient PUC is sustainable. Let $(\mathcal{P}, \pi)$ be an efficient PUC with $\mathcal{P}=\left(p_{1}, \ldots, p_{K}\right)$ and $\pi=\left(C_{1}, \ldots, C_{K}\right)$. Efficiency immediately implies the inequality

$$
\sum_{k=1}^{K^{\prime}} v\left(\Lambda, C_{k}^{\prime}\right) \geq \sum_{k=1}^{K} v\left(\Lambda, C_{k}\right)
$$

for any partition $\pi^{\prime}=\left(C_{1}^{\prime}, C_{2}^{\prime}, \ldots, C_{K^{\prime}}^{\prime}\right)$ of $I$. By (??), this yields

$$
V^{\Lambda}(I)=\sum_{k=1}^{K} v\left(\Lambda, C_{k}\right)
$$

Let $\zeta$ be a core allocation of the game $V^{\Lambda}$, the existence of which is guaranteed by Corollary 4.3. For every $k=1, \ldots, K$ and $t \in C_{k}$ define

$$
x^{k}(t)=\zeta(t)-d\left(\left|t-p^{k}\right|\right)+\alpha
$$

We show first that, for each $k=1, \ldots, K, x^{k}$ is a $C_{k^{-}}$cost allocation. It suffices to demonstrate that

$$
\int_{C_{k}} \zeta(t) \Lambda(t)=g+\int_{C_{k}} d\left(\left|t-p^{k}\right|\right) d \Lambda(t) \quad\left(=v\left(\Lambda, C_{k}\right)\right)
$$

since this clearly implies the budget constraint (??) on $x^{k}$. To this end, consider the measure given by $\mu(T)=\int_{T} \zeta(t) d \Lambda(t)$. Since $\zeta$ is a core allocation, the measure $\mu$ is in the core of $V^{\Lambda}$. Therefore $\mu\left(C_{k}\right) \leq v\left(\Lambda, C_{k}\right)$ for any $k$, and since

$$
\sum_{k=1}^{K} \mu\left(C_{j}\right)=\mu(I)=V^{\Lambda}(I)=\sum_{k=1}^{K} v\left(\Lambda, C_{k}\right),
$$

it follows that $\mu\left(C_{k}\right)=v\left(\Lambda, C_{k}\right)$ for all $k$. Thus (??) is satisfied, and so $x^{k}$ is an $C_{k}$-cost allocation.

We claim next that no community $C$ can reject $(\mathcal{P}, \pi)$ given $\left(x^{1}(t), \ldots, x^{K}(t)\right)$. Indeed, suppose, in negation, that there is a community $C$ and a $C$-cost allocation $x$ such that for every $k=1, \ldots, K$ and $t \in C \cap C_{k}$

$$
x^{k}(t)+d\left(\left|t-p^{k}\right|\right)>x(t)+d(|t-p|) .
$$

By (??),

$$
\int_{C} x(t) d \Lambda(t)=G(C) \geq g>0
$$


and so $\Lambda(C)>0$. Thus, by taking an integral with respect to $\Lambda$ of both sides of (??), we get

$$
\sum_{k=1}^{K} \int_{C \cap C_{k}}\left[x^{k}(t)+d\left(\left|t-p^{k}\right|\right)\right] d \Lambda(t)>\sum_{k=1}^{K} \int_{C \cap C_{k}}[x(t)+d(|t-p|)] d \Lambda(t) .
$$

The left-hand side of (??) is equal to

$$
\sum_{k=1}^{K} \int_{C \cap C_{k}}(\zeta(t)+\alpha) d \Lambda(t)=\int_{C} \zeta(t) d \Lambda(t)+\alpha \Lambda(S)
$$

and the right hand side of (??) is equal to

$$
\sum_{k=1}^{K} \int_{C \cap C_{k}} x(t) d \Lambda(t)+\sum_{k=1}^{K} \int_{C \cap C_{k}} d(|t-p|) d \Lambda(t)=
$$

(by using (??))

$$
=G(C)+\int_{C} d(|t-p|) d \Lambda(t) \geq \alpha \Lambda(C)+V^{\Lambda}(C) .
$$

Thus (??) implies that

$$
\mu(C)=\int_{C} \zeta(t) d \Lambda(t)>V^{\Lambda}(C)
$$

contradicting the assumption that the measure $\mu$ is in the core of $V^{\Lambda}$ ). This establishes the sustainability of $(\mathcal{P}, \pi)$.

It remains to show that every sustainable $\operatorname{PUC}(\mathcal{P}, \pi)=\left(\left(p_{1}, \ldots, p_{K}\right),\left(C_{1}, \ldots, C_{K}\right)\right)$ is efficient. Let $x^{1}, x^{2}, \ldots, x^{K}$ be $C_{k}$-cost allocations such that no community can reject $(\mathcal{P}, \pi)$ given $\left(x^{1}(t), \ldots, x^{K}(t)\right)$. To establish efficiency of $(\mathcal{P}, \pi)$, consider a $\mathrm{PUC}\left(\mathcal{P}^{\prime}, \pi^{\prime}\right)$ with $\mathcal{P}^{\prime}=$ $\left(p_{1}^{\prime}, \ldots, p_{K^{\prime}}^{\prime}\right)$ and $\pi^{\prime}=\left(C_{1}^{\prime}, \ldots, C_{K^{\prime}}^{\prime}\right)$. Note that for every $k_{0}$

$$
D\left(C_{k_{0}}^{\prime}, p_{k_{0}}^{\prime}\right)+G\left(C_{k_{0}}^{\prime}\right) \geq \sum_{k=1}^{K} \int_{C_{k_{0}}^{\prime} \cap C_{k}}\left[x^{k}(t)+d\left(\left|t-p_{k}\right|\right)\right] d \Lambda(t) .
$$

Indeed, if this were not the case, there would be a positive $\delta$ such that a measurable function $y$, given on $C_{k_{0}}^{\prime}$ by

$$
y(t)=x^{k}(t)+d\left(\left|t-p_{k}\right|\right)-d\left(\left|t-p_{k_{0}}^{\prime}\right|\right)-\delta
$$

for each $k$ and $t \in C_{k_{0}}^{\prime} \cap C_{k}$, is a $C_{k_{0}}^{\prime}$-cost allocation. Thus

$$
y(t)+d\left(\left|t-p_{k_{0}}^{\prime}\right|\right)<x^{k}(t)+d\left(\left|t-p_{k}\right|\right)
$$


for each $k$ and $t \in C_{k_{0}}^{\prime} \cap C_{k}$, which means that the community $C_{k_{0}}^{\prime}$ can reject $(\mathcal{P}, \pi)$ (via the $C_{k_{0}}^{\prime}$-cost allocation $\left.y\right)$, contrary to the assumption of sustainability of $(\mathcal{P}, \pi)$.

Inequality (9) and the budget constraint (??) imply that

$$
\begin{gathered}
T\left(\mathcal{P}^{\prime}, \pi^{\prime}\right)=\sum_{k_{0}=1}^{K^{\prime}}\left(D\left(C_{k_{0}}^{\prime}, p_{k_{0}}^{\prime}\right)+G\left(C_{k_{0}}^{\prime}\right)\right) \geq \sum_{k_{0}=1}^{K^{\prime}} \sum_{k=1}^{K} \int_{C_{k_{0}}^{\prime} \cap C_{k}}\left[x^{k}(t)+d\left(\left|t-p_{k}\right|\right)\right] d \Lambda(t) \\
\quad=\sum_{k=1}^{K} \int_{C_{k}}\left[x^{k}(t)+d\left(\left|t-p_{k}\right|\right)\right] d \Lambda(t)=\sum_{k=1}^{K}\left(D\left(C_{k}, p_{k}\right)+G\left(C_{k}\right)\right)=T(\mathcal{P}, \pi) .
\end{gathered}
$$

This establishes the efficiency of $(\mathcal{P}, \pi)$.

\section{Appendix}

Our Appendix consists of two parts. In Part A we prove the auxiliary results, stated in Sections 2 and 4, that have been used in the proof of Main Result. In Part B we prove the propositions stated in Section 3.

\subsection{Part A}

We endow the set $\mathcal{M}(X)$ of positive Borel measures with the weak topology of measures, in which a sequence $\left\{\mu_{n}\right\}_{n=1}^{\infty} \subset \mathcal{M}(X)$ converges to $\mu_{0} \in \mathcal{M}(X)$ if and only if for every continuous function $f$ on $X$

$$
\lim _{n \rightarrow \infty} \int_{X} f(t) d \mu_{n}(t)=\int_{X} f(t) d \mu_{0}(t)
$$

For every subset $A$ of $I$ we denote its topological boundary (relative in $I$ ) by $\partial A$ and for any measure $\Lambda \in M(I)$, the "truncated" measure $\Lambda^{A}$ is determined for every $T \subset I$ by $\Lambda^{A}(T)=\Lambda(A \cap T)$. Finally, in what follows let $\Lambda \in M(I)$ be the probability measure corresponding to the distribution function $F$.

To prove the results, we need several lemmas:

Lemma A.1: The following three conditions on $\left\{\mu_{n}\right\}_{n=0}^{\infty} \subset \mathcal{M}(I)$ are equivalent:

(i) $\left\{\mu_{n}\right\}_{n=1}^{\infty}$ converges weakly to $\mu_{0}$;

(ii) $\lim _{n \rightarrow \infty} \mu_{n}([0, t])=\mu_{0}([0, t])$ for every $t$ such that either $\mu_{0}(\{t\})=0$ or $t=1$; 
(iii) $\lim _{n \rightarrow \infty} \mu_{n}(C)=\mu_{0}(C)$ for every $C \subset I$ with $\mu_{0}(\partial C)=0$

Proof: Consider the set $X=I \cup\{2\}$. Given a sequence $\left\{\mu_{n}\right\}_{n=0}^{\infty}$ satisfying either of the above conditions, let $a=\sup _{n} \mu_{n}(I)$. We can assume that $a>0$, since otherwise the claim is trivial. For every $n$ define $Q_{n} \in M(X)$ by

$$
Q_{n}(C)=\left\{\begin{array}{cc}
\frac{1}{a}\left[\mu_{n}(C \cap I)+a-\mu_{n}(I)\right], & \text { if } 2 \in C, \\
\frac{1}{a} \mu_{n}(C) & \text { otherwise. }
\end{array}\right.
$$

Probability measures $\left\{Q_{n}\right\}_{n=0}^{\infty}$ can be extended to the entire real line $R$ in an obvious fashion. Let:

(i') $\left\{Q_{n}\right\}_{n=1}^{\infty}$ converges weakly to $Q_{0}$;

(ii') $\lim _{n \rightarrow \infty} Q_{n}((-\infty, t])=Q_{0}((-\infty, t])$ for every $t$ with $Q_{0}(\{t\})=0$;

(iii') $\lim _{n \rightarrow \infty} Q_{n}(C)=Q_{0}(C)$ for every $C \subset R$ with $Q_{0}(\widetilde{\partial} C)=0$, where $\widetilde{\partial} C$ denotes the boundary of $C$ in $R$.

Note that the pairs of conditions, (i) and (i'), (ii) and (ii'), (iii) and (iii') are equivalent. Since the equivalence of conditions (i'), (ii'), and (iii') follows from Theorem 28.5 of Billingsley (1995), it follows that (i), (ii) and (iii) are equivalent as well.

Note that the equivalence of (i) and (iii) in Lemma A.1, yields the following useful remark:

Remark A.2: Given a finite or countable $A \subset I$, and $\left\{\Lambda_{n}\right\}_{n=0}^{\infty} \subset M(I), \Lambda_{n}^{A}$ converges weakly to $\Lambda_{0}^{A}$ if and only if $\lim _{n \rightarrow \infty} \Lambda_{n}^{A}(\{t\})=\Lambda_{0}^{A}(\{t\})$ for every $t \in A$.

We also utilize:

Lemma A.3: Any sequence $\left\{\mu_{n}\right\}_{n=1}^{\infty} \subset \mathcal{M}(I)$ with $\sup _{n} \mu_{n}(I)<\infty$ has a weakly converging subsequence.

Proof: By Theorem 29.3 of Billingsley (1995), any sequence of measures in $M(I)$ has a weakly convergent subsequence. The proof follows by using the method applied in the proof of Lemma A.1. 
We now introduce convergence of sets and, in particular, convergence of sequences of intervals. We shall say that a sequence of intervals $\left\{I_{n}\right\}_{n=1}^{\infty}$ converges to interval $I_{0}$ if $\left\{I_{n} \backslash I_{0}\right\}_{n=1}^{\infty}$ and $\left\{I_{0} \backslash I_{n}\right\}_{n=1}^{\infty}$ are monotone sequences of sets (i.e., $I_{n+1} \backslash I_{0} \subset I_{n} \backslash I_{0}$ and $I_{0} \backslash I_{n+1} \subset I_{0} \backslash I_{n}$ for every $n$ ), and $\bigcap_{n=1}^{\infty} I_{n} \backslash I_{0}=\bigcap_{n=1}^{\infty} I_{0} \backslash I_{n}=\emptyset$. To exemplify this mode of convergence, denote by $a_{n}$ and $b_{n}$ the left and right endpoints of $I_{n}$, and suppose that the sequences $\left\{a_{n}\right\}_{n=1}^{\infty}$ and $\left\{b_{n}\right\}_{n=1}^{\infty}$ are monotone with $\lim _{n \rightarrow \infty} a_{n}=a_{0}$ and $\lim _{n \rightarrow \infty} b_{n}=b_{0}$. In the case where none of these sequences becomes constant starting from some $n,\left\{I_{n}\right\}_{n=1}^{\infty}$ converges to $I_{0}$ if $I_{0}=[a, b]$ and $\left\{a_{n}\right\}_{n=1}^{\infty}$ is increasing, $\left\{b_{n}\right\}_{n=1}^{\infty}$ is decreasing; or $I_{0}=(a, b)$ and $\left\{a_{n}\right\}_{n=1}^{\infty}$ is decreasing, $\left\{b_{n}\right\}_{n=1}^{\infty}$ is increasing; or $I_{0}=[a, b)$ and both $\left\{a_{n}\right\}_{n=1}^{\infty}$ and $\left\{b_{n}\right\}_{n=1}^{\infty}$ are increasing; or $I_{0}=(a, b]$ and both $\left\{a_{n}\right\}_{n=1}^{\infty}$ and $\left\{b_{n}\right\}_{n=1}^{\infty}$ are decreasing.

In the case where (say) $\left\{a_{n}\right\}_{n=1}^{\infty}$ becomes constant starting from some $n$ and $\left\{b_{n}\right\}_{n=1}^{\infty}$ is decreasing, $\left\{I_{n}\right\}_{n=1}^{\infty}$ can converge to either $(a, b]$ or $[a, b]$. The convergence is to $(a, b]$ if $\left\{I_{n}\right\}_{n=1}^{\infty}$ exclude $a$ (i.e., intervals $I_{n}$ are open from the left) starting from some $n$, and to $[a, b]$ if $\left\{I_{n}\right\}_{n=1}^{\infty}$ include $a$ starting from some $n$.

We also say that a sequence of communities $\left\{C_{n}\right\}_{n=1}^{\infty}$ converges to a community $C_{0}$ if each $C_{n}$ is a union of the same number of disjoint intervals $I_{1}^{n}, \ldots, I_{K}^{n}$, and, possibly after reordering the intervals, each $\left\{I_{j}^{n}\right\}_{n=1}^{\infty}$ converges to $I_{j}^{0}$.

Lemma A.4: Suppose that a sequence $\left\{C_{n}\right\}_{n=1}^{\infty}$ of communities converges to $C_{0}$, and a sequence $\left\{\Lambda_{n}\right\}_{n=1}^{\infty} \subset M(I)$ converges weakly to $\Lambda \in M(I)$ in a way that $\left\{\Lambda_{n}^{A}\right\}_{n=1}^{\infty}$ also converges weakly to $\Lambda^{A}$, where $A$ denotes the set of atoms of $\Lambda$ (that is, $A=\{t \in I$ $\Lambda(\{t\})>0\})$. Then $\left\{\Lambda_{n}^{C_{n}}\right\}_{n=1}^{\infty}$ converges weakly to $\Lambda^{C_{0}}$.

Proof: It can be assumed without loss of generality that every $C_{n}, n=0,1, \ldots$, is an interval. Indeed, since $\Lambda_{n}^{C_{n}}=\sum_{j} \Lambda_{n}^{I_{j}^{n}}$ for intervals $I_{j}^{n}$ whose union is $C_{n}$, it suffices to show convergence of all summands. Furthermore, it suffices to check, by equivalence of (i) and (ii) in Lemma A.1, that for every interval $T=[0, t]$

$$
\lim _{n \rightarrow \infty} \Lambda_{n}\left(T \cap C_{n}\right)=\Lambda\left(T \cap C_{0}\right)
$$


It follows from our assumptions on $\left\{\Lambda_{n}\right\}_{n=1}^{\infty}$ that $\Lambda_{n}^{I \backslash A}=\Lambda_{n}-\Lambda_{n}^{A}$ converges weakly to $\Lambda^{I \backslash A}=\Lambda-\Lambda^{A}$. By non-atomicity of $\Lambda^{I \backslash A}$ and (iii) in Lemma A.1

$$
\lim _{n \rightarrow \infty} \Lambda_{n}^{I \backslash A}\left(T \cap C_{0}\right)=\Lambda^{I \backslash A}\left(T \cap C_{0}\right) .
$$

Also, for any $n_{0}$

$$
\begin{gathered}
0 \leq \lim \sup _{n \rightarrow \infty} \Lambda_{n}^{I \backslash A}\left(\left[\left(C_{n} \backslash C_{0}\right) \cup\left(C_{0} \backslash C_{n}\right)\right] \cap T\right) \leq \\
\leq \lim \sup _{n \rightarrow \infty} \Lambda_{n}^{I \backslash A}\left(\left(C_{n} \backslash C_{0}\right) \cup\left(C_{0} \backslash C_{n}\right)\right) \leq \lim \sup _{n \rightarrow \infty} \Lambda_{n}^{I \backslash A}\left(\left(C_{n_{0}} \backslash C_{0}\right) \cup\left(C_{0} \backslash C_{n_{0}}\right)\right)= \\
=\Lambda^{I \backslash A}\left(\left(C_{n_{0}} \backslash C_{0}\right) \cup\left(C_{0} \backslash C_{n_{0}}\right)\right),
\end{gathered}
$$

where the last equality again follows from (iii) in Lemma A.1. Since the measure $\Lambda^{I \backslash A}$ is countably additive,

$$
\lim _{n_{0} \rightarrow \infty} \Lambda^{I \backslash A}\left(\left(C_{n_{0}} \backslash C_{0}\right) \cup\left(C_{0} \backslash C_{n_{0}}\right)\right)=0,
$$

and so from (??) - (??)

$$
\lim _{n \rightarrow \infty} \Lambda_{n}^{I \backslash A}\left(\left[\left(C_{n} \backslash C_{0}\right) \cup\left(C_{0} \backslash C_{n}\right)\right] \cap T\right)=0 .
$$

This fact and (??) yield

$$
\lim _{n \rightarrow \infty} \Lambda_{n}^{I \backslash A}\left(T \cap C_{n}\right)=\Lambda^{I \backslash A}\left(T \cap C_{0}\right) .
$$

From Remark A.2 it follows easily that

$$
\lim _{n \rightarrow \infty} \Lambda_{n}^{A}\left(T \cap C_{n}\right)=\Lambda^{A}\left(T \cap C_{0}\right) .
$$

This, coupled with (??), yields (??).

Lemma A.5: Suppose that a sequence $\left\{C_{n}\right\}_{n=1}^{\infty}$ of communities converges to $C$, and a sequence $\left\{\Lambda_{n}\right\}_{n=1}^{\infty} \subset M(I)$ converges weakly to $\Lambda \in M(I)$ in a way that $\left\{\Lambda_{n}^{A}\right\}_{n=1}^{\infty}$ also converges weakly to $\Lambda^{A}$, where $A$ denotes the set of atoms of $\Lambda$ (that is, $A=\{t \in I \mid$ $\Lambda(\{t\})>0\})$. Then

$$
\lim _{n \rightarrow \infty} v\left(\Lambda_{n}, C_{n}\right)=v(\Lambda, C) .
$$


Proof: For every $n$ pick some $p\left(\Lambda_{n}, C_{n}\right) \in O P\left(\Lambda_{n}, C_{n}\right)$, and $p(\Lambda, C) \in O P(\Lambda, C)$. We show first that

$$
\limsup _{n \rightarrow \infty} v\left(\Lambda_{n}, C_{n}\right) \leq v(\Lambda, C) .
$$

Indeed, by definition of $p\left(\Lambda_{n}, C_{n}\right)$,

$$
\int_{C_{n}} d\left(\left|t-p\left(\Lambda_{n}, C_{n}\right)\right|\right) d \Lambda_{n}(t) \leq \int_{C_{n}} d(|t-p(\Lambda, C)|) d \Lambda_{n}(t) .
$$

However, from Lemma A.4,

$$
\lim _{n \rightarrow \infty} \int_{C_{n}} d(|t-p(\Lambda, C)|) d \Lambda_{n}(t)=\int_{C} d(|t-p(\Lambda, C)|) d \Lambda(t),
$$

and so (??) holds. We show next that

$$
\lim \inf _{n \rightarrow \infty} v\left(\Lambda_{n}, C_{n}\right) \geq v(\Lambda, C) .
$$

Otherwise, we could find $\left\{n_{k}\right\}_{k=1}^{\infty}$, a subsequence of integers such that

$$
\lim \inf _{k \rightarrow \infty} v\left(\Lambda_{n_{k}}, C_{n_{k}}\right)<v(\Lambda, C)
$$

and yet $\lim _{k \rightarrow \infty} p\left(\Lambda_{n_{k}}, C_{n_{k}}\right)=p$. From Lemma A.4,

$$
\lim _{k \rightarrow \infty} \int_{C_{n_{k}}} d(|t-p|) d \Lambda_{n_{k}}(t)=\int_{C} d(|t-p|) d \Lambda(t) .
$$

Since $\lim _{k \rightarrow \infty} p\left(\Lambda_{n_{k}}, C_{n_{k}}\right)=p$, the functions $d\left(\left|t-p\left(\Lambda_{n_{k}}, C_{n_{k}}\right)\right|\right)$ converge in the supremum norm to the function $d(|t-p|)$, and so form (??)

$$
\lim _{k \rightarrow \infty} \int_{C_{n_{k}}} d\left(\left|t-p\left(\Lambda_{n_{k}}, C_{n_{k}}\right)\right|\right) d \Lambda_{n_{k}}(t)=\int_{C} d(|t-p|) d \Lambda(t) .
$$

By definition of $p(\Lambda, C)$,

$$
\int_{C} d(|t-p|) d \Lambda(t) \geq \int_{C} d(|t-p(\Lambda, C)|) d \Lambda(t),
$$

and so, from (??),

$$
\lim _{k \rightarrow \infty} v\left(\Lambda_{n_{k}}, C_{n_{k}}\right) \geq v(\Lambda, C),
$$


a contradiction, showing that (??) holds. Thus,

$$
\lim \sup _{n \rightarrow \infty} v\left(\Lambda_{n}, C_{n}\right) \leq v(\Lambda, C) \leq \lim \inf _{n \rightarrow \infty} v\left(\Lambda_{n}, C_{n}\right)
$$

which implies (??).

Lemma A.6: There exists a connected partition $\left(I_{1}, \ldots, I_{K}\right)$ of the set $I$ such that

$$
V^{\Lambda}(I)=\inf \sum_{j=1}^{K} v\left(\Lambda, I_{j}\right)
$$

That is, the infimum in (5) is actually attained for some connected partition of $I$.

\section{Proof: Denote}

$$
V_{c o n}^{\Lambda}(I)=\inf \sum v\left(\Lambda, I_{j}\right)
$$

where the infimum is taken over all finite partitions $\left(I_{1}, \ldots, I_{K}\right)$ of $I$ into disjoint intervals. We shall demonstrate first that this infimum is attained for some connected partition of $I$. Indeed, there is a sequence $\left\{\left(I_{1}^{n}, \ldots, I_{K(n)}^{n}\right)\right\}_{n=1}^{\infty}$ of partitions of $I$ into disjoint intervals such that

$$
\lim _{n \rightarrow \infty} \sum_{j=1}^{K(n)} v\left(\Lambda, I_{j}^{n}\right)=V_{c o n}^{\Lambda}(I) .
$$

Note that $K(n)$ is actually bounded across all $n$, since $g>0$. From compactness of $I$ and the fact that any sequence of numbers has a monotone subsequence it follows that a subsequence $\left\{n_{k}\right\}_{k=1}^{\infty}$ of integers can be found such that $K\left(n_{k}\right) \equiv K$ and $\left\{\inf I_{j}^{n_{k}}\right\}_{k=1}^{\infty}$ and $\left\{\sup I_{j}^{n_{k}}\right\}_{k=1}^{\infty}$ are converging monotone sequences. Thus, each $I_{j}^{n_{k}}$ converges to $I_{j}$, which is either an interval or the empty set; note that the nonempty intervals $I_{j}$ form a partition of $I$. From Lemma A.5 it follows that $\lim _{k \rightarrow \infty} v\left(\Lambda, I_{j}^{n_{k}}\right)=v\left(\Lambda, I_{j}\right)$ provided $I_{j}$ is nonempty, which implies that

$$
V_{c o n}^{\Lambda}(I) \geq \lim \inf _{k \rightarrow \infty} \sum_{j=1}^{K} v\left(\Lambda, I_{j}^{n_{k}}\right) \geq \sum_{I_{j} \neq \emptyset} v\left(\Lambda, I_{j}\right) \geq V_{c o n}^{\Lambda}(I),
$$

and so

$$
\sum_{I_{j} \neq \emptyset} v\left(\Lambda, I_{j}\right)=V_{c o n}^{\Lambda}(I)
$$


It remains to show that $V^{\Lambda}(I)=V_{c o n}^{\Lambda}(I)$. Take a finite set of projects $p_{1}, \ldots, p_{K} \in I$ and consider a partition $\pi$ of $I$ into disjoint communities $C_{1}, \ldots, C_{K}$. Denote by $I_{1}, \ldots, I_{n}$ all the intervals whose unions constitute the communities in $\pi$. Let $\Gamma_{0}$ be the set of $I_{j}$ with $\Lambda\left(I_{j}\right)=0$. Now we perform the following procedure. First, if for some $k$ and $l p_{k}=p_{l}$, unite $C_{k}$ and $C_{l}$. Second, if $I_{j} \subset C_{k}$ but

$$
\int_{I_{j}} d\left(\left|t-p_{k}\right|\right)>\int_{I_{j}} d\left(\left|t-p_{l}\right|\right)
$$

remove the interval $I_{j}$ from $C_{k}$ and attach it to $C_{l}$, and continue the process as long as it is possible. Since the number of intervals is finite, the process will be terminated after a finite number of changes. Denote by $T_{1}, \ldots, T_{K}$ the resulting communities, after removal of $\Gamma_{0}$; some of them might be empty. Note that the convex hull of each $T_{j}$ is disjoint from any other community $T_{l}$. Intervals from $\Gamma_{0}$ can now be added to all $T_{j}$ in the way that will make them convex, but still mutually disjoint. Thus, there are disjoint intervals $T_{1}^{\prime}, \ldots, T_{K}^{\prime}$ (some of them may be empty) that partition $I$ in such a way that

$$
\sum_{j=1}^{K} \int_{T_{j}^{\prime}} d\left(\left|t-p_{j}\right|\right) d \Lambda(t) \leq \sum_{j=1}^{K} \int_{C_{j}} d\left(\left|t-p_{j}\right|\right) d \Lambda(t)
$$

Now, given a partition $\left(C_{1}, \ldots, C_{K}\right)$ of $I$ and $p_{k} \in O P\left(\Lambda, C_{k}\right)$ for every $k=1, \ldots, K$, the above argument shows that there is a connected partition $\left(I_{1}, \ldots, I_{K^{\prime}}\right)$ of $I$ such that

$$
\sum_{j=1}^{K^{\prime}} v\left(\Lambda, I_{j}\right) \leq \sum_{j=1}^{K^{\prime}}\left[\int_{I_{j}} d\left(\left|t-p_{j}\right|\right) d \Lambda(t)+g\right] \leq \sum_{j=1}^{K}\left[\int_{C_{j}} d\left(\left|t-p_{j}\right|\right) d \Lambda(t)+g\right]=\sum_{j=1}^{K} v\left(\Lambda, C_{j}\right)
$$

and so

$$
V_{c o n}^{\Lambda}(I) \leq \inf \sum v\left(\Lambda, C_{j}\right)
$$

Since the opposite inequality is obvious, the proposition is established.

Lemma A.7: If the measures $\left\{\Lambda_{n}\right\}_{n=1}^{\infty}$ and $\Lambda$ are as in Lemma A.5, then

$$
\lim _{n \rightarrow \infty} V^{\Lambda_{n}}(I)=V^{\Lambda}(I) .
$$


Proof: We show first that

$$
\lim \sup _{n \rightarrow \infty} V^{\Lambda_{n}}(I) \leq V^{\Lambda}(I) .
$$

Indeed, by Lemma A.6, there is a partition $\left(I_{1}, \ldots, I_{K}\right)$ of $I$ into disjoint intervals such that

$$
V^{\Lambda}(I)=\sum_{i=1}^{K} v\left(\Lambda, I_{j}\right)
$$

By definition of $V^{\Lambda_{n}}(I)$,

$$
V^{\Lambda_{n}}(I) \leq \sum_{i=1}^{K} v\left(\Lambda_{n}, I_{j}\right)
$$

and hence by Lemma A.5

$$
\lim \sup _{n \rightarrow \infty} V^{\Lambda_{n}}(I) \leq \lim _{n \rightarrow \infty} \sum_{i=1}^{K} v\left(\Lambda_{n}, I_{j}\right)=\sum_{i=1}^{K} v\left(\Lambda, I_{j}\right)=V^{\Lambda}(I) .
$$

Therefore (21) is established.

Now we show that

$$
\lim \inf _{n \rightarrow \infty} V^{\Lambda_{n}}(I) \geq V^{\Lambda}(I) .
$$

Suppose that, on the contrary,

$$
\lim \inf _{n \rightarrow \infty} V^{\Lambda_{n}}(I)<V^{\Lambda}(I)
$$

and that

$$
V^{\Lambda_{n}}(I)=\sum_{i=1}^{K(n)} v\left(\Lambda_{n}, I_{j}^{n}\right)
$$

for every $n$ and some interval partitions $\left(I_{1}^{n}, \ldots, I_{K(n)}^{n}\right)$ of $I$. As in the proof of Lemma A.6 we can find a subsequence $\left\{n_{k}\right\}_{k=1}^{\infty}$ of integers such that $K\left(n_{k}\right) \equiv K$, each $I_{j}^{n_{k}}$ converges to $I_{j}$ which is either an interval or an empty set, and the nonempty intervals $I_{j}$ form a partition of I. From Lemma A.5 it follows that $\lim _{k \rightarrow \infty} v\left(\Lambda_{n_{k}}, I_{j}^{n_{k}}\right)=v\left(\Lambda, I_{j}\right)$ provided $I_{j}$ is nonempty, which implies that

$$
\lim \inf _{k \rightarrow \infty} V^{\Lambda_{n_{k}}}(I)=\lim \inf _{k \rightarrow \infty} \sum_{i=1}^{K} v\left(\Lambda_{n_{k}}, I_{j}^{n_{k}}\right) \geq \sum_{I_{j} \neq \emptyset} v\left(\Lambda, I_{j}\right) \geq V^{\Lambda}(I) .
$$

This contradicts (22). Thus,

$$
\lim \sup _{n \rightarrow \infty} V^{\Lambda_{n}}(I) \leq V^{\Lambda}(I) \leq \lim \inf _{n \rightarrow \infty} V^{\Lambda_{n}}(I)
$$


and so (20) holds.

The following is a corollary of Lemmas A.5 and A.7.

Corollary A.8: For $\left\{\Lambda_{n}\right\}_{n=1}^{\infty}$ and $\Lambda$ as in Lemma A.5, we have $\lim _{n \rightarrow \infty} V^{\Lambda_{n}}(C)=V^{\Lambda}(C)$ for every community $C$.

We now prove the assertion of Proposition 4.1 in the case where the cumulative distribution $F$ has a finite support:

Lemma A.9: If $S \subset I$ is a finite set and $\Lambda \in M(I)$ is supported on $S$ (that is, $\Lambda^{S}=\Lambda$ ), then the game $V^{\Lambda}$ possesses a nonempty core.

Proof: For any subset $T$ of $S$ pick a point $p_{T} \in O P(\Lambda, T)$, and consider the set $F$ consisting of the chosen points. For $t \in S$ and $p \in F$ denote $c_{p t}=\Lambda(\{t\}) \cdot d(|t-p|)$. For any $T \subset S$ define

$$
u(T)=\min \left(g \sum_{p \in F} y_{p}+\sum_{p \in F, t \in T} c_{p t} z_{p t}\right),
$$

where the minimum is taken over all $z_{p t}$ and $y_{p}$ subject to

$$
\begin{gathered}
\sum_{p \in F} z_{p t}=1 \text { for all } t \in T, \\
y_{p}-z_{p t} \geq 0 \text { for all } p \in F, t \in T, \\
y_{p}, z_{p t} \in\{0,1\} \text { for all } p \in F, t \in T .
\end{gathered}
$$

The game $u$ (defined on subsets of $S$ ) represents a minimum cost solution to a facility location problem, described as follows. $F$ is thought of as the set of possible facility locations and $T \subset S$ as a set of customers using these facilities. The cost of transportation to facility $p$ incurred by customer $t$ is given by $c_{p t}$, and the cost of opening any facility $p$ is given by $g$. The variable $y_{p}$ is 1 if facility $p$ is open, and 0 otherwise; the variable $z_{p t}$ is 1 if customer $t$ is connected to facility $p$ and 0 otherwise. Constraints (??) ensure that every customer is connected to exactly one facility. A customer can only be connected to facility that is open, 
by constraints (??). By Theorem 4.2 of Goemans and Skutella (2000), the finite game $u$ has a nonempty core, i.e., there is $\mu \in \mathcal{M}(S)$ such that $\mu(T) \leq u(T)$ for every $T \subset S$, with $\mu(S)=u(S)$

The game $u$ can be extended for any community $C$, by $u(C)=u(C \cap S)$, and the same applies to the measure $\mu$; it is clear that extended $\mu$ is in the core of the extended $u$. It is easy to check that for any community $C \subset I$

$$
u(C)=u(C \cap S) \leq V^{\Lambda}(C \cap S)=V^{\Lambda}(C)
$$

and that $u(I)=u(S)=V^{\Lambda}(S)=V^{\Lambda}(I)$. Thus, the measure $\mu$ is also in the core of the game $V^{\Lambda}$.

Now we turn to our propositions.

Proof of Proposition 2.2: Let $\pi=\left(I_{1}, \ldots, I_{K}\right)$ be a connected partition of $I$ in which the infimum in the definition of $V(\Lambda, I)$ (see (??)) is attained; such a partition exists by Lemma A.6. Also let $p_{k}$ be the $I_{k}$-optimal project for every $k$, and put $\mathcal{P}=\left(p_{1}, \ldots, p_{K}\right)$. From definitions of $V$ and $T, T(\mathcal{P}, \pi)=V^{\Lambda}(I)+\alpha$, and $T\left(\mathcal{P}^{\prime}, \pi^{\prime}\right) \geq V^{\Lambda}(I)+\alpha$ for every other PUC $\left(\mathcal{P}^{\prime}, \pi^{\prime}\right)$. This shows that $\pi$ is, indeed, an efficient connected partition.

Proof of Proposition 4.1: Given $\Lambda \in M(I)$, consider the set of its atoms, $A$. Since $A$ is at most countable, there is an increasing sequence $\left\{A_{n}\right\}_{n=1}^{\infty}$ of finite sets such that $A_{n} \subset A$ and $\bigcup_{n=1}^{\infty} A_{n}=A$. For any $n$, consider the measure $\Lambda_{n}^{\prime}$, supported on the set $\left\{\frac{1}{n}, \frac{2}{n}, \ldots, 1\right\}$, and defined there by

$$
\Lambda_{n}^{\prime}\left(\left\{\frac{i}{n}\right\}\right)=\Lambda^{I \backslash A}\left(\left[\frac{i-1}{n}, \frac{i}{n}\right]\right)
$$

Also consider a measure $\Lambda_{n}^{\prime \prime}$ defined by $\Lambda_{n}^{\prime \prime}=\frac{\Lambda(A)}{\Lambda\left(A_{n}\right)} \Lambda^{A_{n}}$ if $A$ is nonempty, and $\Lambda_{n}^{\prime \prime}=0$ otherwise. Denote $\Lambda_{n}=\Lambda_{n}^{\prime}+\Lambda_{n}^{\prime \prime}$. Thus each measure $\Lambda_{n}$ is supported on the finite set $S_{n}=\left\{\frac{1}{n}, \frac{2}{n}, \ldots, 1\right\} \cup$ $A_{n}$. By checking (ii) of Lemma A.1 and using Remark A.2, it is easy to see that $\Lambda_{n}$ converges to $\Lambda$, and $\Lambda_{n}^{A}$ converges to $\Lambda^{A}$, in the weak topology. 
By Lemma A.9, for each $n$ we can find a measure $\mu_{n}$ in the core of $V^{\Lambda_{n}}$. The measures $\left\{\mu_{n}\right\}_{n=1}^{\infty}$ have a uniformly bounded total mass, since

$$
\lim _{n \rightarrow \infty} \mu_{n}(I)=\lim _{n \rightarrow \infty} V^{\Lambda_{n}}(I)=V^{\Lambda}(I)
$$

The second equality is due to Lemma A.7, and thus, by Lemma A.3, the sequence has a weakly convergent subsequence, which for notational simplicity is assumed to be the sequence itself. Denote the limiting measure in $\mathcal{M}(I)$ by $\mu$. The rest of the proof is dedicated to showing that $\mu$ is in the core of the game $V^{\Lambda}$.

We know that $\mu_{n}(C) \leq V^{\Lambda_{n}}(C)$ for every community $C$, with $V^{\Lambda_{n}}(I)=\mu(I)$. By Lemma A. 1

$$
\lim _{n \rightarrow \infty} \mu_{n}(C)=\mu(C)
$$

for all communities $C$ with $\mu(\partial C)=0$; since the relative boundary of $I$ in itself is an empty set, (??) holds in particular for $C=I$. By Corollary A.8, $\lim _{n \rightarrow \infty} V^{\Lambda_{n}}(C)=V^{\Lambda}(C)$. This shows that

$$
\mu(C) \leq V^{\Lambda}(C)
$$

for all communities $C$ with $\mu(\partial C)=0$; equality holds instead of inequality if $C=I$.

Now let us take a community $C \neq I$ with $\mu(\partial C)>0$ and disjoint intervals $I_{1}, \ldots, I_{K}$ such that $C=\bigcup_{j=1}^{K} I_{j}$. Observe that for any interval $I^{\prime} \subset I$

$$
\partial I^{\prime}=\left\{\begin{array}{cc}
\left\{\inf I^{\prime}, \sup I^{\prime}\right\}, & \text { if } 0,1 \notin I^{\prime}, \\
\left\{\sup I^{\prime}\right\} & \text { if } 0 \in I^{\prime} \text { and } 1 \notin I^{\prime}, \\
\left\{\inf I^{\prime}\right\} & \text { if } 1 \in I^{\prime} \text { and } 0 \notin I^{\prime}, \\
\emptyset & \text { if } I^{\prime}=I .
\end{array}\right.
$$

Thus, if $I_{j}^{\varepsilon, \delta^{1}, \delta^{2}}=\left[\inf I_{j}+\varepsilon \cdot \delta^{1}, \sup I_{j}+\varepsilon \cdot \delta^{2}\right] \cap I$ with $\delta^{1}, \delta^{2} \in\{-1,1\}$, then for almost every $\varepsilon \mu\left(\partial I_{j}^{\varepsilon, \delta^{1}, \delta^{2}}\right)=0$. Therefore (??) holds for $C^{\varepsilon, \delta^{1}, \delta^{2}}=I_{1}^{\varepsilon, \delta_{1}^{1}, \delta_{1}^{2}} \cup \ldots \cup I_{K}^{\varepsilon, \delta_{K}^{1}, \delta_{K}^{2}}$ for almost every $\varepsilon$ and every choice of $\delta^{1}, \delta^{2} \in\{-1,1\}^{k}$, since $\partial C^{\varepsilon, \delta^{1}, \delta^{2}} \subset \bigcup_{j=1}^{K} \partial I_{j}^{\varepsilon, \delta_{j}^{1}, \delta_{j}^{2}}$. It follows that there is a sequence $\left\{\varepsilon_{n}\right\}_{n=1}^{\infty}$ converging to zero such that (??) holds for every $C^{\varepsilon_{n}, \delta^{1}, \delta^{2}}$; clearly $\left\{C^{\varepsilon_{n}, \delta^{1}, \delta^{2}}\right\}_{n=1}^{\infty}$ converges to $C=\bigcup_{j=1}^{K} I_{j}$ for a suitable choice of $\delta^{1}, \delta^{2} \in\{-1,1\}^{k}$. By countable additivity of $\mu$, Lemma A.5, and the fact that $C \neq I$,

$$
\mu(C)=\lim _{n \rightarrow \infty} \mu\left(C^{\varepsilon_{n}, \delta^{1}, \delta^{2}}\right) \leq \lim _{n \rightarrow \infty} V^{\Lambda}\left(C^{\varepsilon_{n}, \delta^{1}, \delta^{2}}\right)=\lim _{n \rightarrow \infty} v\left(\Lambda, C^{\varepsilon_{n}, \delta^{1}, \delta^{2}}\right)=v(\Lambda, C)=V^{\Lambda}(C)
$$


Therefore (??) is satisfied for any community $C$. As we have mentioned, (??) holds as equality for $C=I$, which completes the proof of the proposition.

Proof of Proposition 4.2: According to Proposition 4.1, there is a measure $\mu$ in the core of $V^{\Lambda}$. By Theorem $\mathrm{C}$ in Halmos (1988), there is a Borel set $T \subset I$ such that $\Lambda(T)=0$ and the measure $\mu^{I \backslash T}$ is absolutely continuous with respect to the measure $\Lambda$. Now define a measure $\nu \in \mathcal{M}(I)$ by $\nu=\mu^{I \backslash T}+\mu(T) \cdot \Lambda$. It is clear that $\nu$ is absolutely continuous with respect to $\Lambda$, and we will show that $\nu$ is also in the core of $V^{\Lambda}$.

Consider a community $C \neq I$. The measurability of $C \cup T$ implies that there is a sequence of communities, $\left\{C_{n}\right\}_{n=1}^{\infty} \subset I$, such that

$$
\lim _{n \rightarrow \infty} \Lambda\left(\left[C_{n} \backslash(C \cup T)\right] \cup\left[(C \cup T) \backslash C_{n}\right]\right)=0
$$

and

$$
\lim _{n \rightarrow \infty} \mu\left(\left[C_{n} \backslash(C \cup T)\right] \cup\left[(C \cup T) \backslash C_{n}\right]\right)=0
$$

(e.g., use Theorem D in Halmos (1988) for the measure $\Lambda+\mu$ ). From (??) and the fact that $\Lambda(T)=0$ it follows that

$$
\lim _{n \rightarrow \infty} \Lambda\left(\left[C_{n} \backslash C\right] \cup\left[C \backslash C_{n}\right]\right)=0 .
$$

This implies that for any interval $[0, t]$

$$
\lim _{n \rightarrow \infty} \Lambda\left(C_{n} \cap[0, t]\right)=\Lambda(C \cap[0, t])
$$

and therefore $\left\{\Lambda^{C_{n}}\right\}_{n=1}^{\infty}$ converges weakly to $\Lambda^{C}$, by Lemma A.1. Thus, as in the proof of Lemma A.5, it follows that

$$
\lim _{n \rightarrow \infty} v\left(\Lambda, C_{n}\right)=v(\Lambda, C)
$$

And, from (??),

$$
\lim _{n \rightarrow \infty} \mu\left(C_{n}\right)=\mu(C \cup T) .
$$

Since $\mu$ is in the core of $V^{\Lambda}$, we have $\mu\left(C_{n}\right) \leq v\left(\Lambda, C_{n}\right)$ for any $n$. Using (??) and (??), we obtain $\mu(C \cup T) \leq v(\Lambda, C)$. 
Therefore $\nu(C)=\nu(C \cup T) \leq \mu(C \cup T) \leq v(\Lambda, C)=V^{\Lambda}(C)$, and thus $\nu$ satisfies core equalities for all communities different from $I$. However, $\nu(I)=\mu(I)=V^{\Lambda}(I)$, and so $\nu$ is in the core of the game $V^{\Lambda}$.

\subsection{Part B}

Before proceeding with the proofs of results in Section 3, it would be useful to introduce the minimal aggregate transportation cost of the set $I$ when it is partitioned into a given number of communities. As in Lemma A.6, one can show that this minimum exists and is attained at a connected partition. Thus, for any positive integer $K$, let

$$
D_{K}=\min \sum_{k=1}^{K} D\left(I_{k}^{K}\right)
$$

where the minimum is taken over all partitions $\pi_{K}=\left(I_{1}^{K}, \ldots, I_{K}^{K}\right)$ of $I$ into $K$ intervals.

We now provide a partial characterization of efficient PUC:

Lemma B.1: Suppose that the distribution $F$ possesses a strictly positive density function $f$. Let $(\mathcal{P}, \pi)$ be a PUC such that $\pi=\left(I_{1}, \ldots, I_{K}\right)(K \geq 2)$ is connected and naturally ordered (that is, for every $k<n$, if $t_{k} \in I_{k}$ and $t_{n} \in I_{n}$ then $t_{k}<t_{n}$.) and every $p_{k}$ is $I_{k}$-optimal. Suppose that the minimum in (??) is attained at $\pi$ and the function $f$ is continuous in the neighborhood of the end points of all intervals $I_{k}$. If, $b_{k}=\sup I_{k-1}=$ $\inf I_{k}$, then $b_{k}-p_{k-1}=p_{k}-b_{k}$.

Proof: Denote $a=\inf I_{k-1}, b=b_{k}$, and $c=\sup I_{k}$. The following functions on $[a, c]$,

$$
g(y)=\min _{p \in[a, y]} \int_{[a, y]} d(|t-p|) f(t) d t\left(=\min _{p \in[a, c]} \int_{[a, y]} d(|t-p|) f(t) d t\right)
$$

and

$$
h(y)=\min _{p \in[y, c]} \int_{[y, c]} d(|t-p|) f(t) d t\left(=\min _{p \in[a, c]} \int_{[y, c]} d(|t-p|) f(t) d t\right),
$$

are continuously differentiable in the neighborhood of $b$ and, by the envelope theorem,

$$
g^{\prime}(b)=d\left(b-p_{k-1}\right) f(b), h^{\prime}(b)=-d\left(p_{k}-b\right) f(b) .
$$


By the choice of $\left(I_{1}, \ldots, I_{K}\right), g(y)+h(y)$ attains its maximum over $[a, c]$ at the point $b$, and thus

$$
d\left(b-p_{k-1}\right) f(b)=g^{\prime}(b)=-h^{\prime}(b)=d\left(p_{k}-b\right) f(b)
$$

Since $f$ is positive, the above equality yields $b-p_{k-1}=p_{k}-b$.

The next result provides an asymptotic estimate of aggregate transportation costs incurred by large partitions, and may be of independent interest.

Lemma B.2: Suppose that the distribution $F$ possesses a strictly positive and continuous density function $f$, and that $d$ is differentiable at zero. Then

$$
\lim _{K \rightarrow \infty} K \cdot D_{K}=\frac{d^{\prime}(0)}{4}\left(\int_{0}^{1} \sqrt{f(t)} d t\right)^{2}
$$

Proof: We will first establish (??) under assumption that there is an integer $n$ such that $f$ is a strictly positive function on $I$, equal to a constant $f_{m}$ on each interval of the form $\left[\frac{m}{n}, \frac{m+1}{n}\right)$, and that $d$ is a linear function. We do not assume at this stage that $f$ is a density function. Without loss of generality the slope of $d, d^{\prime}(0)$, is positive, since otherwise the equality (??) is trivial.

Let $\pi_{K}=\left(I_{1}^{K}, \ldots, I_{K}^{K}\right)$ be a partition of $I$ at which the minimum in (??) is attained. Note, using Lemma B.1 and the fact that an $I_{k}^{K}$-optimal project is the midpoint of $I_{k}^{K}$, that any two intervals $I_{k}^{K}$ have the same length if their closures belong to the interior of some $\left[\frac{m}{n}, \frac{m+1}{n}\right)$. Denote by $\Gamma_{m}$ the set of intervals $I_{k}^{K}$ that "fall" into $\left[\frac{m}{n}, \frac{m+1}{n}\right)$ in the above sense, let $a_{m}^{K}$ be the cardinality of $\Gamma_{m}$.

We claim next that the maximal length of an interval in $\pi_{K}$ is bounded by $\frac{c}{K}$ for a constant $c$. Indeed, if not, then there is $c(K) \rightarrow \infty$ such that the maximal length is $\frac{c(K)}{K}$ on a subsequence of $K$. Thus there is $h>0$ such that every element of $\pi_{K}$ in some $\left[\frac{m}{n}, \frac{m+1}{n}\right)$ has the length $\frac{h c(K)}{K}$ on this subsequence. Therefore

$$
D_{K}=\sum_{k=1}^{K} D\left(I_{k}^{K}\right) \geq \min f \sum_{k=1}^{K} d^{\prime}(0) \frac{\left(l\left(\left(I_{k}^{K}\right)\right)\right)^{2}}{4} \geq \frac{\frac{1}{n}-2 \frac{c(K)}{K}}{\frac{c(K)}{K}} d^{\prime}(0) \frac{\min f}{4} K\left(\frac{h c(K)}{K}\right)^{2}
$$


where $l(\cdot)$ stands for the length of the interval. This is bounded from below by $\frac{H c(K)}{K}$ for some constant $H>0$ and all large enough $K$. The following inequality, however, contradicts the existence of this lower bound:

$$
D_{K} \leq \sum_{k=1}^{K-1} D\left(\left[\frac{k-1}{K}, \frac{k}{K}\right)\right)+D\left(\left[\frac{K-1}{K}, 1\right]\right) \leq \sum_{k=1}^{K} \frac{d^{\prime}(0) \max f}{K^{2}}=\frac{d^{\prime}(0) \max f}{K} .
$$

We conclude from the previous paragraph that

$$
\lim \inf _{K \rightarrow \infty} \frac{a_{m}^{K}}{K}>0, \lim _{K \rightarrow \infty} \sum_{m=1}^{n} \frac{a_{m}^{K}}{K}=1
$$

and that for every $m$ there is a sequence $\left\{\varepsilon_{K}^{m}\right\}_{K=1}^{\infty}$ converging to zero, such that the total length of all $I_{k}^{K} \in \Gamma_{m}$ exceeds $\frac{1}{n}-\varepsilon_{K}^{m}$. Since for $I_{k}^{K} \in \Gamma_{m}$

$$
D\left(I_{k}^{K}\right)=d^{\prime}(0) f_{m} \frac{\left(l\left(I_{k}^{K}\right)\right)^{2}}{4} \geq \frac{d^{\prime}(0) f_{m}}{4}\left(\frac{\frac{1}{n}-\varepsilon_{K}^{m}}{a_{m}^{K}+2}\right)^{2}
$$

we have

$$
D_{K}=\sum_{k=1}^{K} D\left(I_{k}^{K}\right) \geq \sum_{m=1}^{n} \frac{d^{\prime}(0) f_{m} a_{m}^{K}}{4}\left(\frac{\frac{1}{n}-\varepsilon_{K}^{m}}{a_{m}^{K}+2}\right)^{2}
$$

and so

$$
K \cdot D_{K} \geq \sum_{m=1}^{n} \frac{K d^{\prime}(0) f_{m} a_{m}^{K}}{4}\left(\frac{\frac{1}{n}-\varepsilon_{K}^{m}}{a_{m}^{K}+2}\right)^{2} .
$$

Since the function $g\left(\beta_{1}, \ldots, \beta_{n}\right)=\sum_{m=1}^{n} \frac{d^{\prime}(0) f_{m}}{4 n^{2} \beta_{m}}$ attains its minimum on the unit simplex $\left(\right.$ where $\left.\sum_{m=1}^{n} \beta_{m}=1\right)$ at $\left(\beta_{1}^{0}, \ldots, \beta_{n}^{0}\right)=\left(\frac{\sqrt{f_{1}}}{\sum_{m=1}^{n} \sqrt{f_{m}}}, \ldots, \frac{\sqrt{f_{n}}}{\sum_{m=1}^{n} \sqrt{f_{m}}}\right)$, it follows from $(? ?)$ and (??) that

$\lim \inf _{K \rightarrow \infty} K \cdot D_{K} \geq \lim \inf _{K \rightarrow \infty} \sum_{m=1}^{n} \frac{K d^{\prime}(0) f_{m} a_{m}^{K}}{4}\left(\frac{\frac{1}{n}-\varepsilon_{K}^{m}}{a_{m}^{K}+2}\right)^{2}=\lim \inf _{K \rightarrow \infty} \sum_{m=1}^{n} \frac{d^{\prime}(0) f_{m}}{4 n^{2} \frac{a_{m}^{K}}{K}} \geq \frac{d^{\prime}(0)}{4} \sum_{m=1}^{n} \frac{f_{m}}{n^{2} \beta_{m}^{0}}$

$$
=\frac{d^{\prime}(0)}{4} \sum_{m=1}^{n} \frac{\sqrt{f_{m}}\left(\sum_{m=1}^{n} \sqrt{f_{m}}\right)}{n^{2}}=\frac{d^{\prime}(0)}{4}\left(\sum_{m=1}^{n} \frac{\sqrt{f_{m}}}{n}\right)^{2}=\frac{d^{\prime}(0)}{4}\left(\int_{0}^{1} \sqrt{f(t)} d t\right)^{2} .
$$

Thus

$$
\lim \inf _{K \rightarrow \infty} K \cdot D_{K} \geq \frac{d^{\prime}(0)}{4}\left(\int_{0}^{1} \sqrt{f(t)} d t\right)^{2} .
$$

Now consider a sequence of integers $\left\{\bar{a}_{m}^{K}\right\}_{K=1}^{\infty}$ for each $m$, such that $\lim _{K \rightarrow \infty} \frac{\bar{a}_{m}^{K}}{K}=\beta_{m}^{0}$, and there are partitions $\left\{\bar{\pi}_{K}\right\}_{K=1}^{\infty}$ of $I$ in which each $\left[\frac{m}{n}, \frac{m+1}{n}\right)$ is partitioned into $\bar{a}_{m}^{K}$ equal intervals $\bar{I}_{k}^{K}$. Since

$$
D_{K}=\sum_{k=1}^{K} D\left(I_{k}^{K}\right) \leq \sum_{k=1}^{K} D\left(\bar{I}_{k}^{K}\right)=\sum_{m=1}^{n} \frac{d^{\prime}(0) f_{m}}{4 n^{2} \bar{a}_{m}^{K}}
$$


and

$$
\begin{aligned}
& \lim _{K \rightarrow \infty} \sum_{m=1}^{n} \frac{K d^{\prime}(0) f_{m}}{4 n^{2} \bar{a}_{m}^{K}}=\lim _{K \rightarrow \infty} \sum_{m=1}^{n} \frac{d^{\prime}(0) f_{m}}{4 n^{2} \frac{\bar{a}_{m}^{K}}{K}}=\sum_{m=1}^{n} \frac{d^{\prime}(0) f_{m}}{4 n^{2} \beta_{m}^{0}} \\
= & \frac{d^{\prime}(0)}{4} \sum_{m=1}^{n} \frac{\sqrt{f_{m}}\left(\sum_{m=1}^{n} \sqrt{f_{m}}\right)}{n^{2}}=\frac{d^{\prime}(0)}{4}\left(\int_{0}^{1} \sqrt{f(t)} d t\right)^{2}
\end{aligned}
$$

it follows that

$$
\lim \sup _{K \rightarrow \infty} K \cdot D_{K} \leq \frac{d^{\prime}(0)}{4}\left(\int_{0}^{1} \sqrt{f(t)} d t\right)^{2}
$$

Using this and (??) we finally obtain

$$
\lim _{K \rightarrow \infty} K \cdot D_{K}=\frac{d^{\prime}(0)}{4}\left(\int_{0}^{1} \sqrt{f(t)} d t\right)^{2} .
$$

Next, we consider a distribution with a strictly positive and continuous density function $f$, while maintaining the assumption that $d$ is linear. By the continuity of $f$, for each $n$ there is a positive function $h_{n}^{+}$which is constant on every interval of the form $\left[\frac{m}{n}, \frac{m+1}{n}\right)$, and

$$
0<h_{n}^{-}(t)=h_{n}^{+}(t)-\varepsilon(n)<f(t)<h_{n}^{+}(t)
$$

for some $\varepsilon(n)$ converging to 0 . For any interval $J$ let $D(J)$ be defined with respect to $h_{n}^{+}$ and $h_{n}^{-}$(and denoted by $D^{h_{n}^{+}}(J)$ and $D^{h_{n}^{-}}(J)$ ), and similarly for $D_{K}^{h_{n}^{+}}$and $D_{K}^{h_{n}^{-}}$. Since clearly $D^{h_{n}^{-}}(J) \leq D(J) \leq D^{h_{n}^{+}}(J)$ for every interval $J$, it follows that

$$
K \cdot D_{K}^{h_{n}^{-}} \leq K \cdot D_{K} \leq K \cdot D_{K}^{h_{n}^{+}}
$$

By applying (??) for $K \cdot D_{K}^{h_{n}^{-}}, K \cdot D_{K}^{h_{n}^{+}}$, and letting $n \rightarrow \infty$, we obtain (??).

Finally, we show that (??) holds even if $d$ is not linear. The differentiability of $d$ at zero implies that for any $\varepsilon>0$ there is an $l>0$ such that for all intervals $J$ with $l(J) \leq l$

$$
D^{\varepsilon^{-}}(J) \leq D(J) \leq D^{\varepsilon+}(J)
$$

where $D^{\varepsilon-}(J)$ is defined with respect to the transportation cost function given by $d^{\varepsilon-}(x)=$ $\max \left\{\left(d^{\prime}(0)-\varepsilon\right) x, 0\right\}$, and $D^{\varepsilon+}(J)$ is defined with respect to $d^{\varepsilon+}(x)=\left(d^{\prime}(0)+\varepsilon\right) x$. But the maximal length of an interval in $\pi_{K}$ converges to zero as $K \rightarrow \infty$, since otherwise 
$\liminf _{K \rightarrow \infty} D_{K}>0$, which is impossible (by taking the partition of $I$ into $K$ equal intervals, the aggregate transportation cost can be made vanishingly small, as in (??)). Therefore

$$
K \cdot D_{K}^{\varepsilon-} \leq K \cdot D_{K} \leq K \cdot D_{K}^{\varepsilon+}
$$

for all large enough $K\left(D_{K}^{\varepsilon-}\right.$ and $D_{K}^{\varepsilon+}$ are defined as in (??), given the definitions of $D^{\varepsilon^{-}}(J)$, $\left.D^{\varepsilon+}(J)\right)$. The equality (??) for $K \cdot D_{K}$ is now obtained by applying it for $K \cdot D_{K}^{\varepsilon-}$ and $K \cdot D_{K}^{\varepsilon+}$, and letting $\varepsilon \rightarrow 0$.

Proof of Proposition 3.1: The proof will be carried out in two parts. In Part I (??) will be established for $K_{g}(I)$ (三 $K_{g}$ ). In Part II we will prove (??) for any interval $J$.

Part I. First, note that $K_{g} \rightarrow \infty$ as $g \rightarrow 0$. Indeed, if $K_{g} \rightarrow \infty$ there is a sequence $\left\{g_{n}\right\}_{n=1}^{\infty}$ that converges to zero and such that $K_{g_{n}} \equiv K$. It follows that for $\pi_{g_{n}}=\left(I_{1}^{g_{n}}, \ldots, I_{K_{g_{n}}}^{g_{n}}\right)$

$$
\lim \inf _{n \rightarrow \infty} \sum_{k=1}^{K\left(g_{n}\right)} D\left(I_{k}^{g_{n}}\right)>0
$$

and so

$$
\lim \inf _{n \rightarrow \infty} \sum_{k=1}^{K\left(g_{n}\right)}\left(D\left(I_{k}^{g_{n}}\right)+G\left(I_{k}^{g_{n}}\right)\right)>0 .
$$

However, we can consider a sequence $\left\{(m(n)\}_{n=1}^{\infty}\right.$ of integers such that $\lim _{n \rightarrow \infty} g_{n} m(n)=0$ and $\lim _{n \rightarrow \infty} m(n)=\infty$, and partitions $\pi_{n}^{\prime}=\left(I_{1}^{\prime}, \ldots, I_{m(n)}^{\prime}\right)=\left(\left[0, \frac{1}{m(n)}\right),\left[\frac{1}{m(n)}, \frac{2}{m(n)}\right), \ldots,\left[\frac{m(n)-1}{m(n)}, 1\right]\right)$. Clearly $\lim _{n \rightarrow \infty} \sum_{k=1}^{m(n)}\left(D\left(I_{k}^{\prime}\right)+G\left(I_{k}^{\prime}\right)\right)=0$, and so, from the definition of $\left\{\pi_{g(n)}\right\}_{n=1}^{\infty}$ as optimal partitions,

$$
\lim \inf _{n \rightarrow \infty} \sum_{k=1}^{K\left(g_{n}\right)}\left(D\left(I_{k}^{g_{n}}\right)+G\left(I_{k}^{g_{n}}\right)\right) \leq \lim _{n \rightarrow \infty} \sum_{k=1}^{m(n)}\left(D\left(I_{k}^{\prime}\right)+G\left(I_{k}^{\prime}\right)\right)=0
$$

contradicting (??).

Let $\varepsilon \in(0,1)$. From the definition of $K_{g}$,

$$
D_{K_{g}}+K_{g} g+\alpha \leq D_{\left[(1+\varepsilon) K_{g}\right]}+\left[(1+\varepsilon) K_{g}\right] g+\alpha
$$

(where $[n]$ stands for the integer part of $n$ ), and so $\varepsilon K_{g} g \geq D_{K_{g}}-D_{\left[(1+\varepsilon) K_{g}\right]}$. Therefore,

$$
\sqrt{\varepsilon K_{g}^{2} g} \geq \sqrt{K_{g} \cdot D_{K_{g}}-\frac{K_{g}}{\left[(1+\varepsilon) K_{g}\right]}\left(\left[(1+\varepsilon) K_{g}\right] \cdot D_{\left[(1+\varepsilon) K_{g}\right]}\right)}
$$


Since $\lim _{g \rightarrow 0} K_{g}=\infty$, by Lemma B.2,

$$
\lim \inf _{g \rightarrow 0} K_{g} \sqrt{g} \geq \frac{1}{2} \frac{\sqrt{d^{\prime}(0)}}{\sqrt{1+\varepsilon}} \int_{0}^{1} \sqrt{f(t)} d t .
$$

But $\varepsilon \in(0,1)$ was arbitrary, and so

$$
\lim \inf _{g \rightarrow 0} K_{g} \sqrt{g} \geq \frac{\sqrt{d^{\prime}(0)}}{2} \int_{0}^{1} \sqrt{f(t)} d t .
$$

Similarly, using the inequality

$$
D_{K_{g}}+K_{g} g+\alpha \leq D_{\left[(1-\varepsilon) K_{g}\right]}+\left[(1-\varepsilon) K_{g}\right] g+\alpha
$$

it can be shown that

$$
\lim _{g \rightarrow \infty} K_{g} \sqrt{g} \leq \frac{\sqrt{d^{\prime}(0)}}{2} \int_{0}^{1} \sqrt{f(t)} d t
$$

which finally yields

$$
\lim _{g \rightarrow \infty} K_{g} \sqrt{g}=\frac{\sqrt{d^{\prime}(0)}}{2} \int_{0}^{1} \sqrt{f(t)} d t .
$$

Part II. For any $g$ consider the minimal interval $J_{g}$ which contains the given $J$ and is composed of intervals in $\pi_{g}$. Since $K_{g}\left(J_{g}\right)-2 \leq K_{g}(J) \leq K_{g}\left(J_{g}\right)$, it suffices to show that

$$
\lim _{g \rightarrow 0} K_{g}\left(J_{g}\right) \sqrt{g}=\frac{\sqrt{d^{\prime}(0)}}{2} \int_{J} \sqrt{f(t)} d t .
$$

From the efficiency of $\pi_{g}$ it follows that $\left.\pi_{g}\right|_{J_{g}}$ (i.e., the set of intervals in $\pi_{g}$ which belong to $\left.J_{g}\right)$ is an efficient partition of $J_{g}$. That is, a $J_{g}$-PUC $\left(\mathcal{P}_{g} \cap J_{g},\left.\pi_{g}\right|_{J_{g}}\right)$ minimizes the aggregate (transportation and project) cost incurred by $J_{g}$. Also, the intervals $J_{g}$ clearly "shrink" to $J$, since the maximal length of an interval in $\pi_{g}$ converges to zero as $g \rightarrow 0$. Thus, (??) can be proved by using essentially the same arguments as in Lemma B.2 to deduce from it the claim of Part I.

To avoid a redundant repetition, we will not give a formal proof of (??). Instead, we will only indicate its main steps. We note first, as in Part I, that $\lim _{g \rightarrow 0} K_{g}\left(J_{g}\right)=\infty$. Then, we establish an analog of Lemma B.2, that for any function $K(g)$ with positive integer values and $\lim _{g \rightarrow 0} K(g)=\infty$, and any sequence of intervals $J(g)$ which "shrinks" to $J$,

$$
\lim _{g \rightarrow 0} K(g) \cdot D_{K(g)}(J(g))=\frac{d^{\prime}(0)}{4}\left(\int_{J} \sqrt{f(t)} d t\right)^{2} .
$$


(Here, $D_{K}(J)$ is defined as the minimum of $\sum_{k=1}^{K} D\left(I_{k}^{K}\right)$, taken over all partitions $\pi_{K}=$ $\left(I_{1}^{K}, \ldots, I_{K}^{K}\right)$ of $J$ into $K$ intervals. $)$

First, we show that (??) holds for a density function $f$ which is equal to a constant $f_{m}$ on each interval of the form $\left[\frac{m}{n}, \frac{m+1}{n}\right)$, and a linear $d$. That is,

$$
\lim _{g \rightarrow 0} K(g) \cdot D_{K(g)}^{f_{g}}(J(g))=\frac{d^{\prime}(0)}{4}\left(\int_{J} \sqrt{f(t)} d t\right)^{2}
$$

where $D_{K(g)}^{f_{g}}(J(g))$ is defined with respect to the "density" 5 function $f_{g}$ (the value of $f_{g}$ on the $i^{\text {th }}$ element of the partition of $J(g)$ into $n$ equal intervals equals to the value of $f$ on the $i^{\text {th }}$ element of the partition of $J$ into $n$ equal intervals; $f_{g}$ coincides with $f$ outside $\left.J(g)\right)$. The proof of (??) is very similar to the proof of the first part of Lemma B.2. At the next stage, we consider the general density function $f$ and approximate it from above and below by sequences $\left(h_{n}^{+}\right)_{n}$ and $\left(h_{n}^{-}\right)_{n}$, as in the proof of Lemma B.2. Since, for small enough $g,\left(h_{n}^{+}\right)_{g}$ and $\left(h_{n}^{-}\right)_{g}$ also approximate $f$ from above and below,

$$
K(g) \cdot D_{K(g)}^{\left(h_{n}^{-}\right)_{g}}(J(g)) \leq K(g) \cdot D_{K(g)}(J(g)) \leq \cdot D_{K(g)}^{\left(h_{n}^{+}\right)_{g}}(J(g)) .
$$

Therefore, by applying (??) to $K(g) \cdot D_{K(g)}^{\left(h_{n}^{-}\right)_{g}}(J(g))$ and $K(g) \cdot D_{K(g)}^{\left(h_{n}^{+}\right)_{g}}(J(g))$ and letting $n \rightarrow \infty$, we obtain (??). The validity of (??) in the case of general $d$ follows as in the proof of Lemma B.2. Finally, (??) is deduced from (??) (where we take $J(g)=J_{g}$ and $\left.K(g)=K_{g}\left(J_{g}\right)\right)$ just as in Part I.

Proof of Proposition 3.2: As in the previous proof, denote by $J_{g}$ the minimal interval which contains the given $J$ and is composed of intervals in $\pi_{g}$. We will show that $\lim _{g \rightarrow 0} \frac{D_{g}\left(J_{g}\right)}{K_{g}\left(J_{g}\right)} \cdot \frac{1}{g}=1$. This will suffice to establish the proposition, since similar methods imply that $\lim _{g \rightarrow 0} \frac{D_{g}\left(J_{g}^{-}\right)}{K_{g}\left(J_{g}^{-}\right)} \cdot \frac{1}{g}=1$ (where $J_{g}^{-}$is the maximal interval contained in $J$, composed of intervals in $\left.\pi_{g}\right), D_{g}\left(J_{g}^{-}\right) \leq D_{g}(J) \leq D_{g}\left(J_{g}\right)$, and $\lim _{g \rightarrow 0} \frac{K_{g}\left(J_{g}^{-}\right)}{K_{g}\left(J_{g}\right)}=1$.

Note that $D_{g}\left(J_{g}\right)=D_{K_{g}\left(J_{g}\right)}\left(J_{g}\right)$. Thus, it remains to show that $\lim _{g \rightarrow 0} \frac{D_{K_{g}\left(J_{g}\right)}\left(J_{g}\right)}{K_{g}\left(J_{g}\right)} \cdot \frac{1}{g}=1$.

\footnotetext{
${ }^{5}$ The quotation marks indicate that, aside from being positive, $f_{g}$ is not required to satisfy $\int_{I} f_{g}(t) d t=1$.
} 
Using (??) and (??), however, it follows that

$$
\begin{gathered}
\lim _{g \rightarrow 0} \frac{D_{K_{g}\left(J_{g}\right)}\left(J_{g}\right)}{K_{g}\left(J_{g}\right)} \cdot \frac{1}{g}=\lim _{g \rightarrow 0} \frac{K_{g}\left(J_{g}\right) \cdot D_{K_{g}\left(J_{g}\right)}\left(J_{g}\right)}{g \cdot K_{g}\left(J_{g}\right)^{2}} \\
=\frac{\lim _{g \rightarrow 0} K_{g}\left(J_{g}\right) \cdot D_{K_{g}\left(J_{g}\right)}\left(J_{g}\right)}{\left(\lim _{g \rightarrow 0} \sqrt{g} \cdot K_{g}\left(J_{g}\right)\right)^{2}}=\frac{\frac{d^{\prime}(0)}{4}\left(\int_{J} \sqrt{f(t)} d t\right)^{2}}{\left(\frac{\sqrt{d^{\prime}(0)}}{2} \int_{J} \sqrt{f(t)} d t\right)^{2}}=1 .
\end{gathered}
$$

Proof of Proposition 2.3: It is a simple corollary of Lemma B.1.

\section{References}

Alesina, A. and E. Spolaore (1997) "On the number and size of nations", Quarterly Journal of Economics 113, 1027-1056.

Alesina, A., Spolaore, E. and R. Wacziarg (2000) "Economic integration and political disintegration", American Economic Review 90, 1276-1296.

Balinski, M.L. (1965) "Integer programming: methods, uses, computation", Management Science 12, 253-313.

Billingsley, P. (1995). Probability and Measure (third edition). Wiley, New York.

Bolton, P., Roland, G. and E. Spolaore (1996) "Economic theories of the breakup and integration of nations", European Economic Review 40, 697-706.

Casella, A. (1992) "On markets and clubs: economic and political integration of regions with unequal productivity", American Economic Review, Papers and Proceedings 82, 115-121.

Cornuejols, G., Nemhauser, G.L. and L.A. Wolsey (1990) "The uncapacited facility location problem" in Discrete Location Theory, P. Mirchandani and R. Francis, eds., John Wiley and Sons, New York, 119-171.

Cremer, H., De Kerchove, A.M. and J. Thisse (1985) "An economic theory of public facilities in space", Mathematical Social Sciences 9, 249-262.

Cremer, H. and J.J. Laffont (2000) "Public goods with costly access", IDEI Working Paper \#98/00, Université des Sciences Sociales de Toulouse. 
Demange, G. (1994) "Intermediate preferences and stable coalition structures", Journal of Mathematical Economics 23, 45-58.

Einy, E., Moreno, D. and B. Shitovitz (1999) "The core of a class of non-atomic games which arise in economic applications ", International Journal of Game Theory 28, 1-14.

Epstein, L.G. and M. Marinacci (2001) "The core of large differentiable TU games ", Journal of Economic Theory 100, 235-273.

Goemans, M.X. and M. Skutella (2000) "Cooperative facility location games", CORE, mimeo.

Grishukhin, V.P. (1994) "On polynomial solvability conditions for the simplest plant location problem", Translations American Mathematical Society Serie 2, 158, 37-46.

Guesnerie, R. and C. Oddou (1981) "Second best taxation as a game", Journal of Economic Theory 25, 67-91.

Guesnerie, R. and C. Oddou (1987) "Increasing returns to size and their limits", Scandinavian Journal of Economics 90, 259-273.

Haimanko, O., Le Breton, M. and S. Weber "Transfers in a polarized country: bridging the gap between efficiency and stability", CORE, mimeo.

Halmos, P. R. (1988). Measure Theory Springer Verlag, New York.

Ichiishi, T. and S. Weber (1978) "Some theorems on the core of a non-side payment game with a measure set of players", International Journal of Game Theory 7, 95-112.

Jéhiel, P. and S. Scotchmer (2001) "Constitutional rules of exclusion in jurisdiction formation", Review of Economic Studies 68, 393-411.

Kannai, Y. (1969) "Countably additive measures in cores of games", Journal of Mathematical Analysis and Applications 27, 227-240.

Kannai, Y. (1992) "The core and balancedness", Chapter 12 in Handbook of Game Theory, R.J. Aumann and S. Hart, eds., North Holland, Amsterdam, 355-395.

Kolen, A. (1983) "Solving covering problems and the uncapacited plant location problem on trees", European Journal of Operational Research 12, 266-278.

Kolen, A. and A. Tamir (1990) "Covering problems" in Discrete Location Theory, P. Mir- 
chandani and R. Francis, eds., John Wiley and Sons, New York, 263-304.

Konishi, H., Le Breton, M. and S. Weber (1998) "Equilibrium in a finite local public good economy", Journal of Economic Theory 79, 224-244.

Le Breton, M., Owen, G. and S. Weber (1992) "Strongly balanced cooperative games", International Journal of Game Theory 20, 419-427.

Le Breton, M. and S. Weber (1999) "The art of making everybody happy: how to prevent a secession?", CORE and Southern Methodist University, mimeo.

Mas-Colell, A. (1980) "Efficiency and decentralization in the pure theory of public goods", Quarterly Journal of Economics, 94, 625-641.

Schmeidler, D. (1967) "On balanced games with infinitely many players", Hebrew University of Jerusalem, mimeo.

Tamir, A. (1992) "On the core of cost allocation games defined on location problems", Transportation Science 27, 81-86.

Trubin, V.A. (1976) "An effective algorithm for solving the distribution problem in a network in the form of a tree", Soviet Mathematics Doklady 17, 1604-1608.

Weber, S. (1981) "Some results on the weak core of a non-sidepayment game with infinitely many players", Journal of Mathematical Economics 8, 101-111.

Weber, S. and S. Zamir (1985) "Proportional taxation: nonexistence of stable structures in an economy with public good", Journal of Economic Theory 35, 178-185.

Wei, S.J. (1991) "To divide or to unite: a theory of secessions", Mimeo, University of California at Berkeley.

Westhoff (1977) "Existence of equilibria in economies with a local public good", Journal of Economic Theory 14, 84-112.

Wooders, M.H. (1978) "Equilibria, the core, and jurisdictional structures in economies with a local public good", Journal of Economic Theory 18, 328-348.

Wooders, M.H. (1983) "The epsilon core of a large replica game" Journal of Mathematical Economics, 11, 277-300.

Wooders, M.H. and W.R. Zame (1984) "Approximate cores of large games", Econometrica 
52, 1327-1350. 